\title{
Finite calculus formulation for incompressible solids using linear triangles and tetrahedra
}

\author{
Eugenio Oñate ${ }^{1, *, \dagger}$, Jerzy Rojek ${ }^{2}$, Robert L. Taylor ${ }^{3, \ddagger}$ and Olgierd C. Zienkiewicz ${ }^{4, \S}$ \\ ${ }^{1}$ International Center for Numerical Methods in Engineering (CIMNE), Universitat Politécnica de Catalunya \\ (UPC), Gran Capitán s/n, Barcelona 08034, Spain \\ ${ }^{2}$ Institute of Fundamental Technological Research, Polish Academy of Sciences, Warsaw, Poland \\ ${ }^{3}$ Department of Civil and Environmental Engineering, University of California, Berkeley, U.S.A. \\ ${ }^{4}$ University of Wales Swansea, Swansea SA2 8PP, U.K.
}

\begin{abstract}
SUMMARY
Many finite elements exhibit the so-called 'volumetric locking' in the analysis of incompressible or quasi-incompressible problems. In this paper, a new approach is taken to overcome this undesirable effect. The starting point is a new setting of the governing differential equations using a finite calculus (FIC) formulation. The basis of the FIC method is the satisfaction of the standard equations for balance of momentum (equilibrium of forces) and mass conservation in a domain of finite size and retaining higher order terms in the Taylor expansions used to express the different terms of the differential equations over the balance domain. The modified differential equations contain additional terms which introduce the necessary stability in the equations to overcome the volumetric locking problem. The FIC approach has been successfully used for deriving stabilized finite element and meshless methods for a wide range of advective-diffusive and fluid flow problems. The same ideas are applied in this paper to derive a stabilized formulation for static and dynamic finite element analysis of incompressible solids using linear triangles and tetrahedra. Examples of application of the new stabilized formulation to linear static problems as well as to the semi-implicit and explicit 2D and 3D non-linear transient dynamic analysis of an impact problem and a bulk forming process are presented. Copyright (C) 2004 John Wiley \& Sons, Ltd.
\end{abstract}

KEY WORDS: finite calculus; volumetric locking; finite element method; linear triangles; linear tetrahedra; static analysis; dynamic analysis

\section{INTRODUCTION}

Many finite elements exhibit the so-called 'volumetric locking' in the analysis of incompressible or quasi-incompressible problems in fluid and solid mechanics. Situations of this type are usual

\footnotetext{
*Correspondence to: Eugenio Oñate, International Center for Numerical Methods in Engineering (CIMNE), Universitat Politécnica de Catalunya (UPC), Gran Capitán s/n, Barcelona 08034, Spain.

†E-mail: onate@cimne.upc.es

¥Visiting Professor, CIMNE, UPC, Barcelona, Spain.

${ }^{\S}$ Unesco Professor at CIMNE, UPC, Barcelona, Spain.
} 
in the structural analysis of rubber materials, some geomechanical problems and most bulk metal forming processes. Volumetric locking is an undesirable effect leading to incorrect numerical results [1].

Volumetric locking in solids is present in all low-order elements based on the standard displacement formulation. The use of a mixed formulation or a selective integration technique eliminates the volumetric locking in many elements. These methods, however, fail in some elements such as linear triangles and tetrahedra, due to lack of satisfaction of the Babuska-Brezzi conditions [1-3] or alternatively the mixed patch test $[1,4,5]$ not being passed.

Considerable efforts have been made in recent years to develop linear triangles and tetrahedra producing correct (stable) results under incompressible situations. Brezzi and Pitkäranta [6] proposed to extend the equation for the volumetric strain rate constraint for Stokes flows by adding a Laplacian of pressure term. A similar method was derived for quasi-incompressible solids by Zienkiewicz and Taylor [1]. Zienkiewicz et al. [7] have proposed a stabilization technique which eliminates volumetric locking in incompressible solids based on a mixed formulation and a characteristic based split (CBS) algorithm initially developed for fluids [8-10] where a split of the pressure is introduced when solving the transient dynamic equations in time. Extensions of the CBS algorithm to solve bulk metal forming problems have been recently reported by Rojek et al. [11]. Other methods to overcome volumetric locking are based on mixed displacement (or velocity)-pressure formulations using the Galerkin-least-square (GLS) method [12], average nodal pressure [13] and average nodal deformation [14] techniques and sub-grid scale (SGS) methods [15-18].

In this paper a different approach is taken to overcome volumetric locking. The starting point is a new setting of the governing differential equations using a finite calculus (FIC) formulation. The basis of the FIC method is the satisfaction of the equations of balance of momentum and that relating the pressure with the volumetric strain in a domain of finite size. The modified differential equations contain additional non-local terms from standard infinitesimal theory. These terms introduce the necessary stability in the discretized equations to overcome the volumetric locking problem.

The FIC approach has been successfully used to derive stabilized finite element and meshless methods for a wide range of advective-diffusive and fluid flow problems [19-26]. The same ideas were applied in Reference [27] to derive a stabilized formulation for quasi-incompressible and incompressible solids allowing the use of linear triangles and tetrahedra. These ideas are extended in this paper where an enhanced formulation with improved pressure stabilization properties is described.

The content of the paper is the following. First, the basis of the FIC method is given for static quasi-incompressible solid mechanics problems. A stabilized formulation for linear triangles and tetrahedra applicable to the full incompressible limit is derived. The stabilized dynamic formulation is also presented and both semi-implicit and explicit monolithic solution schemes are described. The analogies between the FIC formulation and alternative methods for treating incompressible problems are discussed.

In the last part of the paper some examples of application of the new stabilized formulation to a static problem as well to the 2D and $3 \mathrm{D}$ analysis of an impact problem and a bulk forming situation using linear triangles and tetrahedra are given. 
FINITE CALCULUS FORMULATION

\section{BASIC CONCEPTS OF THE FINITE CALCULUS (FIC) METHOD}

Let us consider the equations of equilibrium in a bar (Figure 1). The equilibrium of forces over a segment of finite size belonging to the bar is

$$
N_{A}-N_{B}=0
$$

where $A$ and $B$ are the end points of a finite size domain of length $d$. In Equation (1) $N_{A}$ and $N_{B}$ represent the values of the axial forces at points $A$ and $B$, respectively.

The axial forces $N_{A}$ and $N_{B}$ can be expressed in terms of values at an arbitrary interior point $C$ by the following Taylor series expansion:

$$
\begin{aligned}
& N_{A}=N_{C}-\left.d_{1} \frac{\mathrm{d} N}{\mathrm{~d} x}\right|_{C}+\left.\frac{d_{1}^{2}}{2} \frac{\mathrm{d}^{2} N}{\mathrm{~d} x^{2}}\right|_{C}+O\left(d_{1}^{3}\right) \\
& N_{B}=N_{C}+\left.d_{2} \frac{\mathrm{d} N}{\mathrm{~d} x}\right|_{C}+\left.\frac{d_{2}^{2}}{2} \frac{\mathrm{d}^{2} N}{\mathrm{~d} x^{2}}\right|_{C}+O\left(d_{2}^{3}\right)
\end{aligned}
$$

Substituting Equations (2) into Equation (1) and neglecting cubic terms in $d_{1}$ and $d_{2}$ gives

$$
\frac{\mathrm{d} N}{\mathrm{~d} x}-\underline{\frac{h}{2} \frac{\mathrm{d}^{2} N}{\mathrm{~d} x^{2}}}=0
$$

where $h=d_{1}-d_{2}$ and all the terms are evaluated at the arbitrary point $C$.

Equation (3) is a finite increment form for the equilibrium equation in the domain $A B$. The underlined term in Equation (3) introduces a non-locality effect in the standard equilibrium equations $(\mathrm{d} N / \mathrm{d} x=0)$. Distance $h$ is the characteristic length of the discrete problem and its value depends on the material properties and the parameters of the discretization method chosen (such as the grid size) [19-26]. Note that for $h \rightarrow 0$ the standard infinitesimal form of the balance equation $(\mathrm{d} N / \mathrm{d} x=0)$ is recovered.

The above process can be extended to derive the differential equations expressing balance of momentum, mass, heat, etc. in a domain of finite size for any problem in mechanics as

$$
r_{i}-\underline{\frac{h_{k}}{2} \frac{\partial r_{i}}{\partial x_{k}}}=0
$$

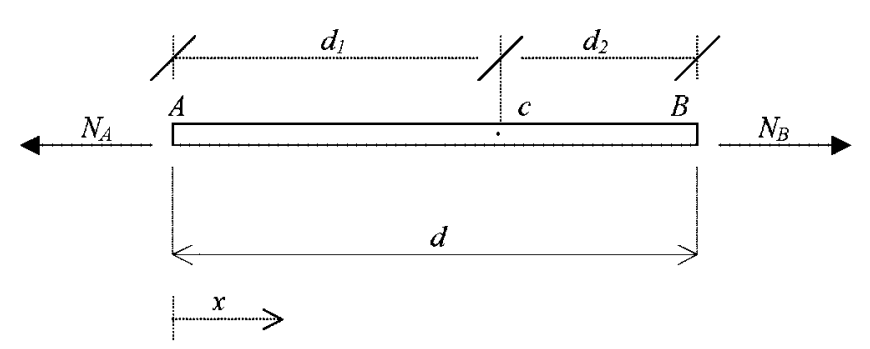

Figure 1. Equilibrium forces in a finite segment of a bar. 
where $r_{i}$ is the standard form of the $i$ th differential equation for the infinitesimal problem, $h_{k}$ are the characteristic lengths of the domain where balance of fluxes, forces, etc. is enforced and $k=1,2,3$ for 3D problems. In Equation (4) and in the following, summation convention for repeated indexes is assumed. Again, note that the underlined terms in Equation (4) can be viewed as non-local forms of the original differential equations. These terms are essential in order to introduce the necessary stabilization for the discrete solution of some problems using whatever numerical technique. Details of the derivation of Equation (4) for steady state and transient advective-diffusive and fluid flow problems can be found in References [19-22]. Applications of the FIC approach to the Galerkin finite element solution of these problems are given in References [23-25]. A meshless method based on the FIC formulation is presented in Reference [26].

The underlined stabilization terms in Equations (3) and (4) are a consequence of accepting that the infinitesimal form of the balance equations is an unreachable limit within the framework of a discrete numerical solution. Indeed Equations (3) and (4) are not useful for obtaining a straightforward analytical solution following traditional integration methods based on infinitesimal calculus theory. The meaning of the new differential equations makes, however, full sense in the context of a discrete numerical method, yielding approximate values of the solution at a finite collection of points within the analysis domain. Convergence to the exact analytical solution value at these points will occur as the grid size tends to zero, which also implies naturally an evolution towards a zero value of the characteristic length parameters.

The FIC procedure has been interpreted in Reference [28] as a general residual correction method where a numerical solution is sought to a modified system of governing differential equations. In the modified equations not only the original residuals but also the derivatives of these residuals multiplied by characteristic length distances appear. A similar intepretation of the FIC procedure as an equation modification method is presented in Reference [29]. A variational setting for the FIC equations in elasticity problems is presented in Reference [30].

\section{FIC FORMULATION FOR INCOMPRESSIBLE ELASTICITY}

\subsection{Equilibrium equations}

Following the arguments of the previous section the equilibrium equations for an elastic solid are written using the FIC technique as [19]

$$
r_{i}-\frac{h_{k}}{2} \frac{\partial r_{i}}{\partial x_{k}}=0 \quad \text { in } \Omega, \quad k=1, n_{\mathrm{d}}
$$

where $n_{\mathrm{d}}$ is the number of space dimensions of the problems (i.e. $n_{\mathrm{d}}=3$ for $3 \mathrm{D}$ )

$$
r_{i}:=\frac{\partial \sigma_{i j}}{\partial x_{j}}+b_{i}
$$

In Equations (5) and (6) $\sigma_{i j}$ and $b_{i}$ are the stresses and the body forces, respectively, and $h_{k}$ are characteristic length distances of an arbitrary prismatic domain where equilibrium of forces is considered. 
Equations (5) and (6) are completed with the boundary conditions on the displacements $u_{i}$

$$
u_{i}-\bar{u}_{i}=0 \quad \text { on } \Gamma_{u}
$$

and the equilibrium of surface tractions

$$
\sigma_{i j} n_{j}-\bar{t}_{i}-\underline{\frac{1}{2} h_{k} n_{k} r_{i}}=0 \quad \text { on } \Gamma_{t}
$$

In the above $\bar{u}_{i}$ and $\bar{t}_{i}$ are prescribed displacements and tractions over the boundaries $\Gamma_{u}$ and $\Gamma_{t}$, respectively, $n_{i}$ are the components of the unit normal vector and $h_{k}$ are again the characteristic lengths.

The form of Equation (8) with the additional 'residual' term underlined is a consequence of expressing the equilibrium of surface tractions in a boundary domain of finite size and retaining higher order terms than those usually accepted in the infinitesimal theory [19].

\subsection{Constitutive equations}

As usual in quasi-incompressible problems the stresses are split into deviatoric and volumetric (pressure) parts

$$
\sigma_{i j}=s_{i j}+p \delta_{i j}
$$

where $\delta_{i j}$ is the Kronnecker delta function. The linear elastic constitutive equations for the deviatoric stresses $s_{i j}$ are written as

$$
s_{i j}=2 G\left(\varepsilon_{i j}-\frac{1}{3} \varepsilon_{v} \delta_{i j}\right)
$$

where $G$ is the shear modulus,

$$
\varepsilon_{i j}=\frac{1}{2}\left(\frac{\partial u_{i}}{\partial x_{j}}+\frac{\partial u_{j}}{\partial x_{i}}\right) \quad \text { and } \quad \varepsilon_{v}=\varepsilon_{i i}
$$

The constitutive equation for the pressure $p$ can be written for an arbitrary domain of finite size as

$$
\frac{1}{K} p^{\mathrm{av}}=\frac{\Delta V}{V}
$$

where $K$ is the bulk modulus of the material and $p^{\text {av }}$ is the average value of the pressure over any arbitrary domain of finite size of volume $V$.

The value of $p^{\text {av }}$ can be approximated as

$$
p^{\mathrm{av}}=\frac{1}{V} \int_{V} p \mathrm{~d} V=p-\frac{h_{k}}{2} \frac{\partial p}{\partial x_{k}}+O\left(h_{k}\right)^{2}
$$

where $p$ is the pressure at an arbitrary point within the domain $V$ and $h_{k}$ are characteristic lengths of such a domain; $h_{k}=d_{1}^{k}-d_{2}^{k}$, where $k=1,2,3$ for 3D and $d_{1}^{k}$ and $d_{2}^{k}$ are the 3D extensions of distances $d_{1}$ and $d_{2}$ in Figure 1.

Similarly, the ratio $\Delta V / V$ can be expressed as

$$
\frac{\Delta V}{V}=\varepsilon_{v}-\frac{h_{k}}{2} \frac{\partial \varepsilon_{v}}{\partial x_{k}}+O\left(h_{k}\right)^{2}
$$


Substituting Equations (13) and (14) into Equation (12) and neglecting second order terms in $h_{k}$ gives the FIC constitutive equation for the pressure as

$$
\left(\frac{1}{K} p-\varepsilon_{v}\right)-\frac{h_{k}}{2} \frac{\partial}{\partial x_{k}}\left(\frac{1}{K} p-\varepsilon_{v}\right)=0, \quad k=1,2,3 \text { for } 3 \mathrm{D}
$$

Note that for $h_{k} \rightarrow 0$ the standard relationship between the pressure and the volumetric strain of the infinitesimal theory $\left(p=K \varepsilon_{v}\right)$ is found.

For an incompressible material $K \rightarrow \infty$ and Equation (15) yields

$$
\varepsilon_{v}-\frac{h_{k}}{2} \frac{\partial \varepsilon_{v}}{\partial x_{k}}=0
$$

Equation (16) expresses the limit incompressible behaviour of the solid. This equation is typical in incompressible fluid dynamic problems there and arises from the mass continuity conditions $[19,20]$.

By combining Equations (5), (6), (9), (10) and (16) a mixed displacement-pressure formulation can be written as

$$
\begin{aligned}
\frac{\partial s_{i j}}{\partial x_{j}}+\frac{\partial p}{\partial x_{i}}+b_{i}-\frac{h_{k}}{2} \frac{\partial r_{i}}{\partial x_{k}} & =0 \\
\left(\frac{p}{K}-\varepsilon_{v}\right)-\frac{h_{k}}{2} \frac{\partial}{\partial x_{k}}\left(\frac{p}{K}-\varepsilon_{v}\right) & =0
\end{aligned}
$$

Substituting Equation (10) into (17) leads after some algebra to

$$
\frac{\partial \varepsilon_{v}}{\partial x_{i}}=\frac{3}{2 G}\left[\hat{r}_{i}-\frac{h_{k}}{2} \frac{\partial r_{i}}{\partial x_{k}}\right]
$$

where $r_{i}$ is defined by Equation (6) and

$$
\hat{r}_{i}=\frac{\partial}{\partial x_{j}}\left(2 G \varepsilon_{i j}\right)+\frac{\partial p}{\partial x_{i}}+b_{i}
$$

Substituting Equation (19) into (18) gives

$$
\left(\frac{p}{K}-\varepsilon_{v}\right)-\frac{h_{i}}{2}\left(\frac{1}{K} \frac{\partial p}{\partial x_{i}}-\frac{3}{2 G} \hat{r}_{i}\right)-\left(\frac{3 h_{i}}{8} \frac{h_{k}}{G} \frac{\partial r_{i}}{\partial x_{k}}\right)=0
$$

Each of the three bracketed terms in Equation (21) is identically zero for the exact analytical solution. This is obvious for the first and third term. For the second term we have that $(1 / K) \partial p / x_{j}=\partial \varepsilon_{v} / x_{j}$ for the exact solution and, hence, in the limit we recover

$$
\frac{1}{K} \frac{\partial p}{\partial x_{i}}-\frac{3}{2 G} \hat{r}_{i}=\frac{\partial \varepsilon_{v}}{\partial x_{i}}-\frac{3}{2 G} \hat{r}_{i}=\frac{-3}{2 G} r_{i}
$$

which vanishes for the exact solution. Consequently, this term will be neglected in the subsequent derivation and only the term involving the derivatives of $r_{i}$ will be retained in Equation (21). Note that this term can take high values in zones where sharp gradients of the numerical solution error occur, despite the fact that the actual value of $r_{i}$ may be relatively low. 
Also, the terms involving products $h_{i} h_{j}$ for $i \neq j$ will be neglected in Equation (21) as they have not been found to contribute to improving the quality of the numerical results.

\subsection{Weighted residual forms}

In conclusion, the following two governing equations will be used for a mixed displacement pressure formulation:

$$
\begin{array}{r}
\frac{\partial s_{i j}}{\partial x_{j}}+\frac{\partial p}{\partial x_{i}}+b_{i}-\frac{h_{k}}{2} \frac{\partial r_{i}}{\partial x_{k}}=0 \\
\left(\frac{p}{K}-\varepsilon_{v}\right)-\sum_{i=1}^{n_{\mathrm{d}}} \tau_{i} \frac{\partial r_{i}}{\partial x_{i}}=0
\end{array}
$$

with the boundary conditions given by Equations (7) and (8) and

$$
\tau_{i}=\frac{3 h_{i}^{2}}{8 G}
$$

The coefficients $\tau_{i}$ in Equation (23b) are also referred to as intrinsic time parameters. Note that the value of $\tau_{i}$ in Equation (24) deduced from the FIC formulation basically coincides for $h_{i}=h_{j}=h$ with that of $\tau=h^{2} / 2 G$ heuristically chosen in other works [6, 14-18].

The weighted residual form of the new governing equations is

\section{Equilibrium:}

$$
\int_{\Omega} \delta u_{i}\left[\frac{\partial s_{i j}}{\partial x_{j}}+\frac{\partial p}{\partial x_{i}}+b_{i}\right] \mathrm{d} \Omega-\int_{\Omega} \delta u_{i} \frac{h_{k}}{2} \frac{\partial r_{i}}{\partial x_{k}} \mathrm{~d} \Omega+\int_{\Gamma_{t}} \delta u_{i}\left[\sigma_{i j} n_{j}-\bar{t}_{i}-\frac{h_{k}}{2} n_{k} r_{i}\right] \mathrm{d} \Gamma=0
$$

Pressure constitutive equation:

$$
\int_{\Omega} q\left(\frac{p}{K}-\varepsilon_{v}\right) \mathrm{d} \Omega-\int_{\Omega} q\left(\sum_{i=1}^{n_{\mathrm{d}}} \tau_{i} \frac{\partial r_{i}}{\partial x_{i}}\right) \mathrm{d} \Omega=0
$$

where $\delta u_{i}$ and $q$ are arbitrary test functions representing virtual displacements and virtual pressure fields, respectively.

Integrating by parts, the terms involving $s_{i j}, p$ and $r_{i}$ in Equation (25a) and the term involving $r_{i}$ in Equation (25b) and neglecting the space derivatives of the characteristic lengths leads to

\section{Equilibrium:}

$$
\int_{\Omega} \delta \varepsilon_{i j}\left(s_{i j}+\delta_{i j} p\right) \mathrm{d} \Omega-\int_{\Omega} \delta u_{i} b_{i} \mathrm{~d} \Omega-\int_{\Gamma_{t}} \delta u_{i} \bar{t}_{i} \mathrm{~d} \Omega-\int_{\Omega} \frac{h_{k}}{2} \frac{\partial \delta u_{i}}{\partial x_{k}} r_{i} \mathrm{~d} \Omega=0
$$

Pressure constitutive equation:

$$
\int_{\Omega} q\left(\frac{p}{K}-\varepsilon_{v}\right) \mathrm{d} \Omega+\int_{\Omega}\left(\sum_{i=1}^{n_{\mathrm{d}}} \frac{\partial q}{\partial x_{i}} \tau_{i} r_{i}\right) \mathrm{d} \Omega-\int_{\Gamma} q \tau_{i} n_{i} r_{i} \mathrm{~d} \Gamma=0
$$


The first three terms in Equation (26a) are the standard in the principle of virtual work in solid mechanics [10]. Note that the term involving $r_{i}$ has vanished from the boundary integrals after the integration by parts. The last integral in Equation (26a) is essential to stabilize the numerical solution in convection-dominated problems [20,24-26]. This term is not relevant for solid mechanics problems and will be omitted hereafter.

Also, the third integral in Equation (26b) along the domain boundary will not be taken into account hereafter as its effect on the stabilization of the pressure equation is negligible.

With these modifications the set of integral equations to be solved are

\section{Equilibrium:}

$$
\int_{\Omega} \delta \varepsilon_{i j}\left(s_{i j}+\delta_{i j} p\right) \mathrm{d} \Omega-\int_{\Omega} \delta u_{i} b_{i} \mathrm{~d} \Omega-\int_{\Gamma_{t}} \delta u_{i} \bar{t}_{i} \mathrm{~d} \Gamma=0
$$

Pressure constitutive equation:

$$
\int_{\Omega} q\left(\frac{p}{K}-\varepsilon_{v}\right) \mathrm{d} \Omega+\int_{\Omega}\left(\sum_{i=1}^{n_{\mathrm{d}}} \frac{\partial q}{\partial x_{i}} \tau_{i} r_{i}\right) \mathrm{d} \Omega=0
$$

\section{Remark}

Note that even though the terms involving the characteristic lengths have been removed from Equation (26a), these terms are essential to provide the necessary stabilization terms in Equation (27b). Consequently, the finite calculus formulation of both the equilibrium and the pressure constitutive equations given by Equations (17) and (18), respectively, is needed.

The key stabilizing term in Equation (27b) is the second integral. From Equations (6) and (9) we can write the expression of $r_{i}$ as

$$
r_{i}=\frac{\partial p}{\partial x_{i}}+\pi_{i}
$$

where

$$
\pi_{i}=\frac{\partial s_{i j}}{\partial x_{j}}+b_{i}
$$

Note that $\pi_{i}$ is the part of $r_{i}$ not containing the pressure gradient and may be interpreted as the negative of a projection of the pressure gradient. In a discrete setting the terms $\pi_{i}$ can be considered as belonging to a sub-scale space orthogonal to that of the pressure gradient terms.

In the infinitesimal limit $r_{i}=0$ and $\left(\partial p / x_{i}\right)+\pi_{i}=0$. This limit relationship between $\partial p / x_{i}$ and $\pi_{i}$ can be weakly enforced by means of the following weighted residual forms:

$$
\int_{\Omega} w_{i} \tau_{i}\left(\frac{\partial p}{\partial x_{i}}+\pi_{i}\right) \mathrm{d} \Omega=0, \quad i=1, n_{\mathrm{d}} ; \text { no sum in } i
$$

where $w_{i}$ are appropriate weighting functions $\left(w_{i} \equiv \delta \pi_{i}\right)$ and the term $\tau_{i}$ is introduced in order to ensure symmetry of the final system of equations. 
With the above assumptions the set of governing equations can be written as

$$
\begin{aligned}
\int_{\Omega} \delta \varepsilon_{i j}\left(s_{i j}+\delta_{i j} p\right) \mathrm{d} \Omega-\int_{\Omega} \delta u_{i} b_{i} \mathrm{~d} \Omega-\int_{\Gamma_{t}} \delta u_{i} \bar{t}_{i} \mathrm{~d} \Gamma & =0 \\
\int_{\Omega} q\left(\frac{p}{K}-\varepsilon_{v}\right) \mathrm{d} \Omega+\int_{\Omega}\left[\sum_{i=1}^{n_{\mathrm{d}}} \frac{\partial q}{\partial x_{i}} \tau_{i}\left(\frac{\partial p}{\partial x_{i}}+\pi_{i}\right)\right] \mathrm{d} \Omega & =0 \\
\int_{\Omega} w_{i} \tau_{i}\left(\frac{\partial p}{\partial x_{i}}+\pi_{i}\right) \mathrm{d} \Omega & =0, \text { no sum in } i
\end{aligned}
$$

with $i, j=1, n_{\mathrm{d}}$.

\section{FINITE ELEMENT DISCRETIZATION}

We will choose $C^{0}$ continuous linear interpolations of the displacements, the pressure and the pressure gradient projection $\pi_{i}$ over three-node triangles (2D) and four-node tetrahedra (3D). The linear interpolations are written as

$$
\begin{gathered}
u_{i}=\sum_{j=1}^{n} N_{j} \bar{u}_{i}^{j} \\
p=\sum_{j=1}^{n} N_{j} \bar{p}^{j} \\
\pi_{i}=\sum_{j=1}^{n} N_{j} \bar{\pi}_{i}^{j}
\end{gathered}
$$

where $n=3(4)$ for $2 \mathrm{D}(3 \mathrm{D})$ problems and $\left(^{(}\right)$denotes nodal variables. As usual $N_{j}$ are the linear shape functions [1].

Substituting approximations (31) into Equations (30) leads to the following system of Galerkin discretized equations (for $\delta u_{i}=q=w_{i}=N_{i}$ ):

$$
\left[\begin{array}{ccc}
\mathbf{A} & \mathbf{G} & \mathbf{0} \\
\mathbf{G}^{\mathrm{T}} & -(\mathbf{C}+\mathbf{L}) & -\mathbf{Q} \\
\mathbf{0} & -\mathbf{Q}^{\mathrm{T}} & -\overline{\mathbf{C}}
\end{array}\right]\left\{\begin{array}{l}
\overline{\mathbf{u}} \\
\overline{\mathbf{p}} \\
\bar{\pi}
\end{array}\right\}=\left\{\begin{array}{l}
\mathbf{f} \\
\mathbf{0} \\
\mathbf{0}
\end{array}\right\}
$$

where the element contributions are given by (for $3 \mathrm{D}$ problems)

$$
\begin{aligned}
& \mathbf{A}_{i j}=\int_{\Omega^{e}} \mathbf{B}_{i}^{\mathrm{T}} \mathbf{D}_{\mathrm{d}} \mathbf{B}_{j} \mathrm{~d} \Omega, \quad \mathbf{G}_{i j}=\int_{\Omega^{e}}\left(\boldsymbol{\nabla} N_{i}\right) N_{j} \mathrm{~d} \Omega \\
& L_{i j}=\int_{\Omega^{e}} \boldsymbol{\nabla}^{\mathrm{T}} N_{i}[\tau] \boldsymbol{\nabla} N_{j} \mathrm{~d} \Omega, \quad C_{i j}=\int_{\Omega^{e}} \frac{1}{K} N_{i} N_{j} \mathrm{~d} \Omega
\end{aligned}
$$




$$
\begin{aligned}
\overline{\mathbf{C}} & =\left[\begin{array}{ccc}
\overline{\mathbf{C}}^{1} & \mathbf{0} & \mathbf{0} \\
\mathbf{0} & \overline{\mathbf{C}}^{2} & \mathbf{0} \\
\mathbf{0} & \mathbf{0} & \overline{\mathbf{C}}^{3}
\end{array}\right], \quad \bar{C}_{i j}^{k}=\int_{\Omega^{e}} \tau_{k} N_{i} N_{j} \mathrm{~d} \Omega \\
\mathbf{Q} & =\left[\mathbf{Q}^{1}, \mathbf{Q}^{2}, \mathbf{Q}^{3}\right], \quad Q_{i j}^{k}=\int_{\Omega^{e}} \tau_{k} \frac{\partial N_{i}}{\partial x_{k}} N_{j} \mathrm{~d} \Omega \\
\mathbf{f}_{i} & =\int_{\Omega^{e}} N_{i} \mathbf{b} \mathrm{d} \Omega+\int_{\Gamma} N_{i} \overline{\mathbf{t}} \mathrm{d} \Gamma, \quad i, j=1, n_{\mathrm{d}}
\end{aligned}
$$

In the above $\mathbf{b}=\left[b_{1}, b_{2}, b_{3}\right]^{\mathrm{T}}$ and $\overline{\mathbf{t}}=\left[\bar{t}_{1}, \bar{t}_{2}, \bar{t}_{3}\right]^{\mathrm{T}}$,

$$
\boldsymbol{\nabla}=\left\{\begin{array}{c}
\frac{\partial}{\partial x_{1}} \\
\frac{\partial}{\partial x_{2}} \\
\frac{\partial}{\partial x_{3}}
\end{array}\right\}, \quad[\tau]=\left[\begin{array}{ccc}
\tau_{1} & & \mathbf{0} \\
& \tau_{2} & \\
\mathbf{0} & & \tau_{3}
\end{array}\right]
$$

$\mathbf{B}$ is the standard infinitesimal strain matrix and $\mathbf{D}_{\mathrm{d}}$ is the deviatoric constitutive matrix [1]. The intrinsic time parameters $\tau_{i}$ are computed by Equation (24). The consistent definition of the characteristic length parameters is still an open question. In advective-diffusive and fluid flow problems it is usual to accept that the characteristic length vector has the direction of the velocity vector (this is the so-called streamline upwind Petrov-Galerkin or SUPG assumption $[26,31,32])$. A method for computing the characteristic lengths in terms of the element residuals for advective-diffusive and fluid flow problems is described in References [21-25]. In the quasistatic example presented in this paper we have obtained good results using a simpler definition of the characteristic lengths with $h_{i}=h_{j}=\left[\Omega^{(\mathrm{e})}\right]^{1 / n_{\mathrm{d}}}$ where $\Omega^{(\mathrm{e})}$ is the element area or volume for $2 \mathrm{D}$ and $3 \mathrm{D}$ problems, respectively. The computation of the intrinsic time parameter for dynamic problems is discussed in Section 6.

There are a number of possibilities for solving Equations (32). The simultaneous solution of Equation (32) involves seven d.o.f per node for 3D problems (three displacements, one pressure and three projected pressure gradient variables). A reduced system of equations can be obtained as follows. Using the last row of Equation (32) the pressure gradient projection variables can be formally expressed in terms of the nodal pressures as

$$
\bar{\pi}=-\overline{\mathbf{C}}^{-1} \mathbf{Q}^{\mathrm{T}} \overline{\mathbf{p}}
$$

Substituting this equation into the second row of Equation (32) leads to the following system of equations in terms of the velocity and pressure variables only:

$$
\left[\begin{array}{cc}
\mathbf{A} & \mathbf{G} \\
\mathbf{G}^{\mathrm{T}} & -(\mathbf{C}+\mathbf{L}-\mathbf{S})
\end{array}\right]\left\{\begin{array}{l}
\overline{\mathbf{u}} \\
\overline{\mathbf{p}}
\end{array}\right\}=\left\{\begin{array}{l}
\mathbf{f} \\
\mathbf{0}
\end{array}\right\}
$$


where

$$
\mathbf{S}=\mathbf{Q} \overline{\mathbf{C}}^{-1} \mathbf{Q}^{\mathrm{T}}
$$

Note that $\mathbf{S}$ is a symmetric matrix as $\overline{\mathbf{C}}$ is symmetric.

For the fully incompressible case $K \rightarrow \infty, \mathbf{C}=\mathbf{0}$ and the resulting system is

$$
\left[\begin{array}{cc}
\mathbf{A} & \mathbf{G} \\
\mathbf{G}^{\mathrm{T}} & -(\mathbf{L}-\mathbf{S})
\end{array}\right]\left\{\begin{array}{l}
\overline{\mathbf{u}} \\
\overline{\mathbf{p}}
\end{array}\right\}=\left\{\begin{array}{l}
\mathbf{f} \\
\mathbf{0}
\end{array}\right\}
$$

The diagonal term $\mathbf{L}-\mathbf{S}$ provides the necessary stability to the system of equations in order to find the nodal values of $\overline{\mathbf{u}}$ and $\overline{\mathbf{p}}$.

The inversion of matrix $\overline{\mathbf{C}}$ can be simplified by considering a lumped matrix $\overline{\mathbf{C}}_{\mathrm{d}}=\operatorname{diag} \overline{\mathbf{C}}$ as an approximation to $\overline{\mathbf{C}}$.

The solution for $\overline{\mathbf{u}}$ and $\overline{\mathbf{p}}$ can also be found iteratively using the previous iteration values of the projected pressure gradient. Starting from Equation (36) the iterative algorithm can be written as

$$
\left[\begin{array}{cc}
\mathbf{A} & \mathbf{G} \\
\mathbf{G}^{\mathrm{T}} & -(\mathbf{C}+\mathbf{L})
\end{array}\right]\left\{\begin{array}{c}
\overline{\mathbf{u}}^{(i)} \\
\overline{\mathbf{p}}^{(i)}
\end{array}\right\}=\left\{\begin{array}{c}
\mathbf{f} \\
\mathbf{Q} \overline{\boldsymbol{\pi}}^{(i-1)}
\end{array}\right\}
$$

with

$$
\overline{\boldsymbol{\pi}}^{(i-1)}=-\overline{\mathbf{C}}^{-1} \mathbf{Q}^{\mathrm{T}} \overline{\mathbf{p}}^{(i-1)}
$$

where again advantage can be taken of a diagonal form of $\mathbf{M}$. This algorithm has been suggested by Chiumenti et al. $[17,18]$, who have proposed a similar formulation for elastic and elastoplastic problems based on the SGS method. As noted in Reference [17] the computational cost due to the iterative algorithm is negligible in a non-linear context where the projected pressure gradient can be computed within the equilibrium iterations induced by the non-linearity.

Note that the algorithm of Equation (39a) is also applicable for the full incompressible case when $K=\infty$ and $\mathbf{C}=0$.

\subsection{Simplification and analogies with other formulations}

It is interesting to note that the form of Equation (36) is very similar to that obtained using the CBS method [7,8,11]. The FIC and CBS forms can be made in fact identical if the time increment in the CBS method is chosen to be coincident with the intrinsic time parameter. Note, however, that the FIC equations have been derived here without the need of a split process.

An analogy can also be found between Equation (36) and the stabilized system of equation resulting from the GLS method. A comparison of the CBS and GLS formulations for incompressible fluid flow problems can be found in Reference [33].

A simpler stabilized formulation can be derived by neglecting the effect of the projected pressure gradient terms. As deduced from Equation (28b) these terms are formally zero for a linear displacement interpolation and the absence of body forces. The resulting system of 
equations for $\overline{\mathbf{u}}$ and $\overline{\mathbf{p}}$ in this case is deduced from Equation (32) as

$$
\left[\begin{array}{cc}
\mathbf{A} & \mathbf{G} \\
\mathbf{G}^{\mathrm{T}} & -(\mathbf{C}+\mathbf{L})
\end{array}\right]\left\{\begin{array}{l}
\overline{\mathbf{u}} \\
\overline{\mathbf{p}}
\end{array}\right\}=\left\{\begin{array}{l}
\mathbf{f} \\
\mathbf{0}
\end{array}\right\}
$$

This formulation was presented by the authors in Reference [24].

An identical form to Equation (40) was obtained by Brezzi and Pitkäranta [6] for Stokes flows and extended to elasticity by Zienkiewicz and Taylor [1]. In both these approaches the weighted form of the standard pressure constitutive equation is augmented with a pressure Laplacian term emanating from the divergence of the momentum equations.

Equation (40) for $[\tau]=\tau \mathbf{I}$ also coincides with the GLS formulation using a linear interpolation for $\mathbf{u}$ and $p$ [33].

The full form of Equation (32) for $[\tau]=\tau \mathbf{I}$ is identical to that derived by Chiumenti et al. [17,18] using a sub-grid approach where the projected pressure gradient $\pi$ is taken as an approximation of the non-resolvable sub-grid scales assumed to be orthogonal to the finite element space. This method was first proposed by Codina $[15,16]$ for the stabilization of convection and incompressibility effects in fluid flow problems.

The formulation proposed here has distinct features from the methods mentioned above:

- The general form of Equation (32) emerges naturally from the governing equations derived using the finite calculus (FIC) formulation.

- The 'stabilization' properties of the discretized equations appear already at the continuum level (Equations (17) and (18)) and they are a consequence of the intrinsic properties of the new governing equations deduced from the FIC method.

- The FIC formulation leads to the identification of the stabilization parameters as a function of the material properties and the characteristic length distances (Equation (24)).

- The effect of the space change in the intrinsic time parameters can be easily taken into account by incorporating the appropriate derivative terms in the integrals of Equations (26).

- A refined FIC method can be considered by incorporating the effect of the last integrals in Equations (26). This can be simply implemented within an iterative solution scheme.

- The FIC approach is readily applicable to any interpolation order for $\mathbf{u}, p$ and $\pi$. The form of the resulting equation system will be identical to Equation (32) (as long as the last two integrals of Equations (26) are still neglected). Application of the FIC method to the formulation of a four-node quadrilateral element with linear interpolation for all variables adequate for quasi and fully incompressible situations is reported in Reference [28].

Note finally that if the element is $\mathrm{BB}$ stable, the introduction of stabilization is not necessary [1].

\section{NON-LINEAR TRANSIENT DYNAMIC FORMULATION}

The static formulation can be readily extended for the transient dynamic case accounting for geometrical and material non-linear effects. Indeed in many situations of this kind, typical of forming processes, impact and crashworthiness problems, among others, material 
quasi-incompressibility develops in specific zones of the solid due to the accumulation of plastic strains. It is well known that in these cases the use of equal order interpolations for displacements and pressure leads to locking solutions unless some precautions are taken. A stabilized finite element formulation based on the CBS method allowing for linear triangles and tetrahedra for transient dynamic analysis of quasi-incompressible solids was reported by the authors in References [7,11]. A similar formulation based on the FIC approach which does not require the split process is described next.

The transient equilibrium equations using the FIC method can be written in an identical form to Equation (5) (neglecting time stabilization terms $[19,24,25]$ ) as

$$
r_{i}-\frac{h_{j}}{2} \frac{\partial r_{i}}{\partial x_{j}}=0
$$

with

$$
r_{i}:=-\rho \frac{\partial^{2} u_{i}}{\partial t^{2}}+\frac{\partial \sigma_{i j}}{\partial x_{j}}+b_{i}
$$

where $\rho$ is the density and $t$ the time.

Equation (41a) is completed with the constitutive equations for the deviatoric stresses (Equation (10)) and the pressure (Equation (15)), as well with the boundary conditions (7) and (8) and the initial conditions for $t=0$.

Following the arguments of the static case, the stabilized constitutive equation for the pressure can be expressed in terms of the residuals of the momentum equations by an expression identical to Equation (23b). This equation is now written in an incremental form more suitable for nonlinear transient analysis.

The set of stabilized equations to be solved are now:

\section{Equilibrium:}

$$
r_{i}-\frac{h_{j}}{2} \frac{\partial r_{i}}{\partial x_{j}}=0
$$

Pressure constitutive equation:

$$
\frac{\Delta p}{K}-\frac{\partial\left(\Delta u_{i}\right)}{\partial x_{i}}-\sum_{i=1}^{n_{\mathrm{d}}} \tau_{i} \frac{\partial r_{i}}{\partial x_{i}}=0
$$

where $\Delta p=p^{n+1}-p^{n}$ and $\Delta u_{i}=u_{i}^{n+1}-u_{i}^{n}$ are the increments of pressure and displacements, respectively. As usual $(\cdot)^{n}$ denotes values at time $t_{n}$ and $\tau_{i}=3 h_{i}^{2} / 8 G$.

In the derivation of Equation (42b) we have accepted that $\Delta r_{i}=r_{i}^{n+1} \equiv r_{i}$ as the infinitesimal equilibrium equations are assumed to be satisfied at time $t_{n}$ (and hence $r_{i}^{n}=0$ ).

Similar to the static case, the residual $r_{i}$ is now split as

$$
r_{i}=\pi_{i}+\frac{\partial p}{\partial x_{i}}
$$


where

$$
\pi_{i}=-\rho \frac{\partial^{2} u_{i}}{\partial t^{2}}+\frac{\partial s_{i j}}{\partial x_{i}}+b_{i}
$$

Following the same steps of the static case the weighted residual form of the governing equations (42a), (42b) and (43a) can be written in the form

$$
\begin{array}{r}
\int_{\Omega} \delta u_{i} \rho \frac{\partial^{2} u_{i}}{\partial t^{2}} \mathrm{~d} \Omega+\int_{\Omega} \delta \varepsilon_{i j} \sigma_{i j} \mathrm{~d} \Omega-\int_{\Omega} \delta u_{i} b_{i} \mathrm{~d} \Omega-\int_{\Gamma} \delta u_{i} \bar{t}_{i} \mathrm{~d} \Gamma=0 \\
\int_{\Omega} q\left(\frac{\Delta p}{K}-\frac{\partial\left(\Delta u_{i}\right)}{\partial x_{i}}\right) \mathrm{d} \Omega+\int_{\Omega}\left[\sum_{i=1}^{n_{\mathrm{d}}} \frac{\partial q}{\partial x_{i}} \tau_{i}\left(\frac{\partial p}{\partial x_{i}}+\pi_{i}\right)\right] \mathrm{d} \Omega=0 \\
\int_{\Omega}\left[\sum_{i=1}^{n_{\mathrm{d}}} w_{i} \tau_{i}\left(\frac{\partial p}{\partial x_{i}}+\pi_{i}\right)\right] \mathrm{d} \Omega=0
\end{array}
$$

The finite element discretization of the displacements, the pressure and the pressure gradient projections is expressed by Equations (31) with the nodal variables now being a function of the time $t$. Substituting approximations (31) into Equations (44) gives the following system of discretized equations:

$$
\begin{array}{r}
\mathbf{M} \ddot{\overline{\mathbf{u}}}+\mathbf{g}-\mathbf{f}=\mathbf{0} \\
\mathbf{G}^{\mathrm{T}} \Delta \overline{\mathbf{u}}-\mathbf{C} \Delta \overline{\mathbf{p}}-\mathbf{L} \overline{\mathbf{p}}-\mathbf{Q} \bar{\pi}=\mathbf{0} \\
\mathbf{Q}^{\mathrm{T}} \overline{\mathbf{p}}+\overline{\mathbf{C}} \bar{\pi}=\mathbf{0}
\end{array}
$$

where $\ddot{\overline{\mathbf{u}}}$ is the nodal acceleration vector,

$$
M_{i j}=\int_{\Omega^{e}} \rho N_{i} N_{j} \mathrm{~d} \Omega
$$

is the mass matrix

$$
\mathbf{g}=\int_{\Omega} \mathbf{B}^{\mathrm{T}} \boldsymbol{\sigma} \mathrm{d} \Omega
$$

is the internal nodal force vector and the rest of the matrices and vectors are defined in Equation (33). Note that the expression of $\mathbf{g}$ of Equation (47) is adequate for non-linear structural analysis.

A four-step semi-explicit time integration algorithm can be derived from Equations (45) as follows:

Step 1: Compute the nodal velocities $\dot{\overline{\mathbf{u}}}^{n+1 / 2}$

$$
\dot{\overline{\mathbf{u}}}^{n+1 / 2}=\dot{\overline{\mathbf{u}}}^{n-1 / 2}+\Delta t \mathbf{M}_{\mathrm{d}}^{-1}\left(\mathbf{f}^{n}-\mathbf{g}^{n}\right)
$$


Step 2: Compute the nodal displacements $\overline{\mathbf{u}}^{n+1}$

$$
\overline{\mathbf{u}}^{n+1}=\overline{\mathbf{u}}^{n}+\Delta t \dot{\overline{\mathbf{u}}}^{n+1 / 2}
$$

Step 3: Compute the nodal pressures $\overline{\mathbf{p}}^{n+1}$

$$
\overline{\mathbf{p}}^{n+1}=[\mathbf{C}+\mathbf{L}]^{-1}\left[\Delta t \mathbf{G}^{\mathrm{T}} \dot{\overline{\mathbf{u}}}^{n+1 / 2}+\mathbf{C} \overline{\mathbf{p}}^{n}-\mathbf{Q} \bar{\pi}^{n}\right]
$$

Step 4: Compute the nodal projected pressure gradients $\bar{\pi}^{n+1}$

$$
\bar{\pi}^{n+1}=-\overline{\mathbf{C}}_{\mathrm{d}}^{-1} \mathbf{Q}^{\mathrm{T}} \overline{\mathbf{p}}^{n+1}
$$

In the above, all matrices are evaluated at $t^{n+1},(\cdot)_{\mathrm{d}}=\operatorname{diag}(\cdot)$ and

$$
\mathbf{g}^{n}=\int_{\Omega^{e}}\left[\mathbf{B}^{\mathrm{T}} \boldsymbol{\sigma}\right]^{n} \mathrm{~d} \Omega
$$

where the stresses $\boldsymbol{\sigma}^{n}$ are obtained by consistent integration of the adequate (non-linear) constitutive law [48].

Note that steps 1, 2 and 4 are fully explicit as a diagonal form of matrices $\mathbf{C}$ and $\overline{\mathbf{C}}$ has been chosen. The solution of step 3 with a diagonal form for $\mathbf{C}$ still requires the inverse of a Laplacian matrix. This can be an inexpensive process using an iterative equation solution method (e.g. a preconditioned conjugate gradient method).

A three-step approach can be obtained by evaluating the projected pressure gradient variables $\bar{\pi}^{n+1}$ at $t_{n+1}$ in a fully implicit form in Equation (48c). Eliminating $\bar{\pi}^{n+1}$ from the fourth step using Equation (48d) and substituting this expression into Equation (48c) leads to

$$
\overline{\mathbf{p}}^{n+1}=[\mathbf{C}+\mathbf{L}-\mathbf{S}]^{-1}\left[\Delta t \mathbf{G}^{\mathrm{T}} \dot{\overline{\mathbf{u}}}^{n+1 / 2}+\mathbf{C} \overline{\mathbf{p}}^{n}\right]
$$

where

$$
\mathbf{S}=\mathbf{Q} \overline{\mathbf{C}}_{\mathrm{d}}^{-1} \mathbf{Q}^{\mathrm{T}}
$$

Recall that for the full incompressible case $K=\infty$ and $\mathbf{C}=0$ in all the above equations.

The critical time step $\Delta t$ is taken as that of the standard explicit dynamic scheme $[10,28]$.

\subsection{Fully explicit algorithm}

A fully explicit four-step algorithm can be obtained by computing $\overline{\mathbf{p}}^{n+1}$ from step 3 in Equation (48c) as follows:

$$
\overline{\mathbf{p}}^{n+1}=\mathbf{C}_{\mathrm{d}}^{-1}\left[\Delta t \mathbf{G}^{\mathrm{T}} \dot{\mathbf{u}}^{n+1 / 2}+\left(\mathbf{C}_{\mathrm{d}}-\mathbf{L}\right) \overline{\mathbf{p}}^{n}-\mathbf{Q} \bar{\pi}^{n}\right]
$$

Elimination of $\overline{\boldsymbol{\pi}}^{n}$ from Equation (48d) leads to the following explicit expression of $\overline{\mathbf{p}}^{n+1}$ for the three-step scheme:

$$
\overline{\mathbf{p}}^{n+1}=\mathbf{C}_{\mathrm{d}}^{-1}\left[\Delta t \mathbf{G}^{\mathrm{T}} \dot{\overline{\mathbf{u}}}^{n+1 / 2}+\left(\mathbf{C}_{\mathrm{d}}-\mathbf{L}+\mathbf{S}\right) \overline{\mathbf{p}}^{n}\right]
$$

Obviously, the solution of Equations (52) breaks down for $K=\infty$ as $\mathbf{C}=0$ in this case. Therefore, the explicit algorithm is not applicable in the full incompressible limit. The explicit 
form can, however, be used with success in problems where quasi-incompressible regions exist adjacent to standard 'compressible' zones. Examples of this kind are shown in the second and third examples of the next section. In both cases the semi-implicit and explicit schemes gave identical results with important savings in both computer time and memory storage requirements obtained when using the explicit form.

\section{ABOUT THE COMPUTATION OF THE INTRINSIC TIME PARAMETER FOR NON-LINEAR TRANSIENT PROBLEMS}

The expression of the intrinsic time parameter is given by $\tau_{i}=3 h_{i}^{2} / 8 G$ (see Equation (24)) where $h_{i}$ are characteristic length parameters and $G$ is the shear modulus. The computation of the characteristic lengths $h_{i}$ is a critical step in stabilized methods. In practice, it is usual to accept that all $h_{i}$ are identical and constant within each element and given by $h_{i}=h^{(\mathrm{e})}=$ $\left[V^{(\mathrm{e})}\right]^{1 / 3}$ where $V^{(\mathrm{e})}$ is the element volume (or the element area for $2 \mathrm{D}$ problems). This expression for $h_{i}$ does not take into account the element distortions along a particular direction during the deformation process.

The correct value of the shear modulus in the expression of $\tau_{i}$ is another sensitive issue as, obviously, for non-linear problems the value of $G$ will differ from the elastic modulus. This fact has been identified by Cervera et al. [34] for non-linear analysis of incompressible problems using linear triangles.

A useful alternative to compute $\tau_{i}$ for explicit non-linear transient situations is to make use of the value of the speed of sound in an elastic solid, defined by

$$
c=\sqrt{\frac{E}{\rho}}
$$

where $E$ is the Young's modulus. The stability condition for explicit dynamic computations is given by the Courant condition defined as [10]

$$
\Delta t^{(\mathrm{e})} \leqslant \Delta t_{\mathrm{c}}^{(\mathrm{e})}=\frac{h^{(\mathrm{e})}}{c}
$$

where $\Delta t_{\mathrm{c}}^{(\mathrm{e})}$ is the critical time step for the element and $h^{(\mathrm{e})}$ is a representative element dimension along the direction of the velocity vector.

Accepting that $G \simeq E / 3$ for the incompressible case and using Equations (24), (53) and (54) (assuming the identify in Equation (54)) an alternative expression for the element intrinsic time parameter in terms of the critical time step can be found as

$$
\tau^{(\mathrm{e})} \stackrel{\left[\left[\Delta t_{\mathrm{c}}^{(\mathrm{e})}\right]^{2}\right.}{\rho}
$$

Equation (55) shows clearly that the intrinsic time parameter varies across the mesh as a function of the critical time step for each element.

Equation (55) is used to compute the intrinsic time parameter for each element in the dynamic examples presented in the next section using both the semi-explicit and the fully explicit forms. 


\section{NUMERICAL RESULTS}

\subsection{Driven cavity}

We consider the problem of a square region in which the top is displaced horizontally by a unit displacement. This is a standard problem in fluid mechanics known as the driven cavity problem in which a velocity is specified at the top instead of a displacement. The cavity has a unit length for both the horizontal and vertical sides. The material properties are Young's modulus, $E$, of 3 and Poisson's ratio, $v$, of 0.49999995 . This gives shear modulus of near unity, a ratio of the bulk to shear modulus $(K / G)$ approximately equal to $10^{7}$ and thus represents a quasi-incompressible solid. To represent the driven cavity all boundaries have zero displacement in both the normal and tangential directions except the top face which has a unit horizontal displacement at all nodes except the corner ones which are set to zero. For the solution reported here the pressure at the centre of the bottom face is set to zero. The problem is solved using different meshes ranging from 10 elements per side to 40 elements per side. The $10 \times 10$ mesh is shown in Figure 2.

To illustrate the effects of the sub-scale $\pi_{i}$ terms we plot the pressure along the horizontal centreline in Figure 3. The curve labelled T1P1 ignores the $\pi_{i}$ terms in all equations and this leads to a form which includes only the displacements $\mathbf{u}$ and the pressure $p$ at each node. In this form the equations are identical to that introduced originally by Brezzi and Pitkäranta [6]. In Figure 4, we show the same result for a $40 \times 40$ mesh. These results have been computed with the monolithic equations (Equation (32)) in which all five degrees of freedom $\left(u_{1}, u_{2}, p, \pi_{1}\right.$ and $\left.\pi_{2}\right)$ are included in a single solution. The value for the intrinsic time parameter is set as $\tau_{i} \equiv \tau=\gamma h^{2} /(2 G)$ where $h^{2}$ is taken as twice the area of each triangle and $\gamma=0.75$ as given by the finite calculus derivation.

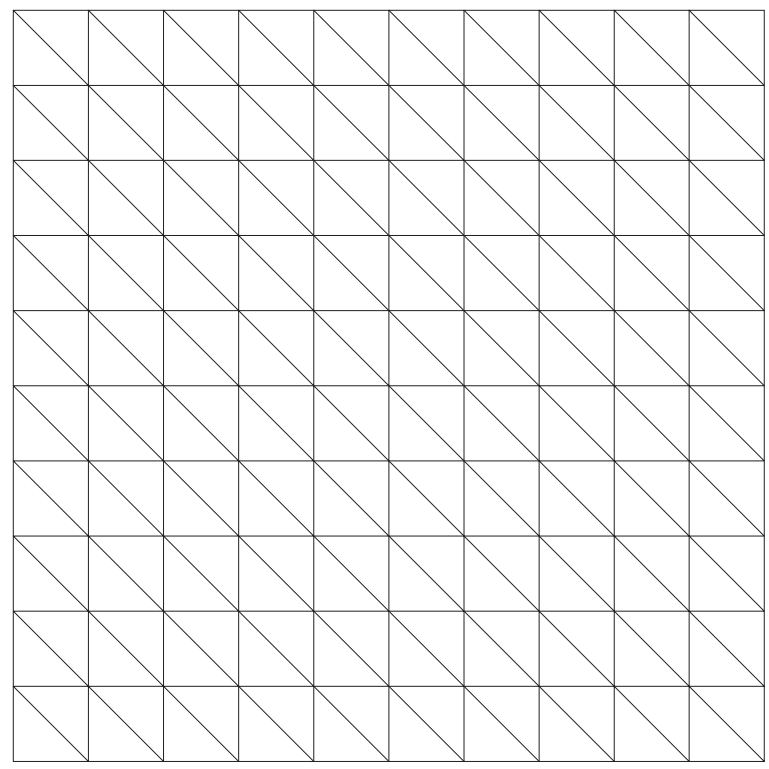

Figure 2. $10 \times 10$ Mesh of triangles. 


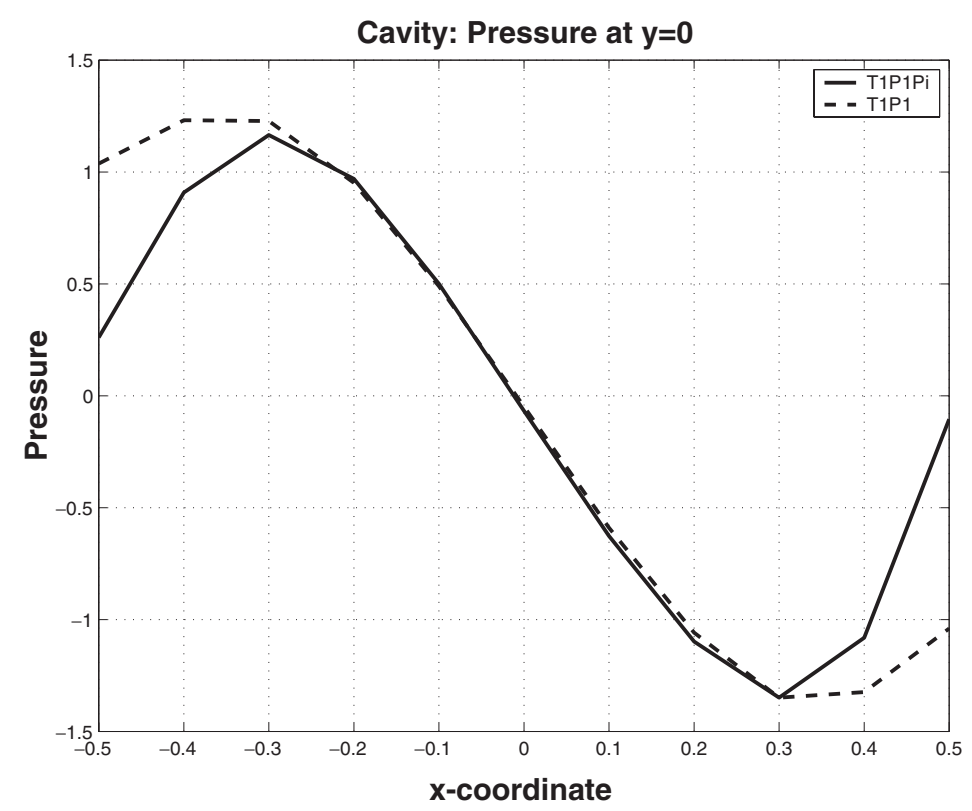

Figure 3. Pressure on horizontal centreline, $10 \times 10$ mesh.

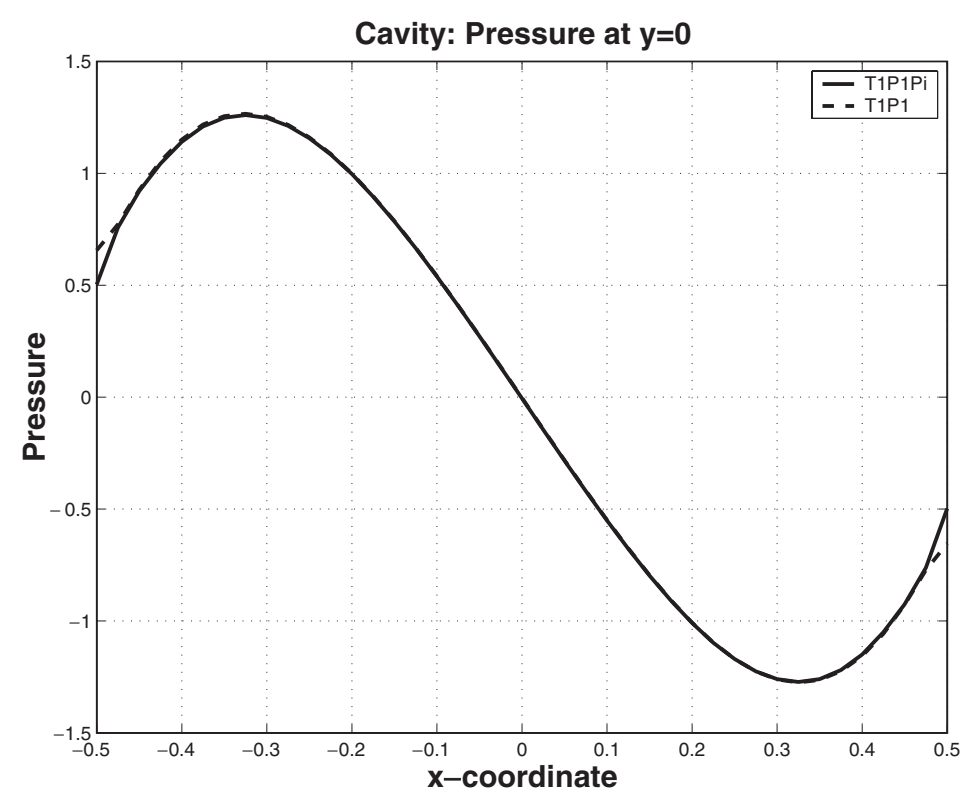

Figure 4. Pressure on horizontal centreline, $40 \times 40$ mesh.

The above solution was repeated using the iterative solution in which the displacement and pressure variables are solved separately from the $\pi_{i}$ variables. Figures 5 and 6 show the results for iterations 1,3 and 5. The result for the $10 \times 10$ mesh was iterated to convergence and the 


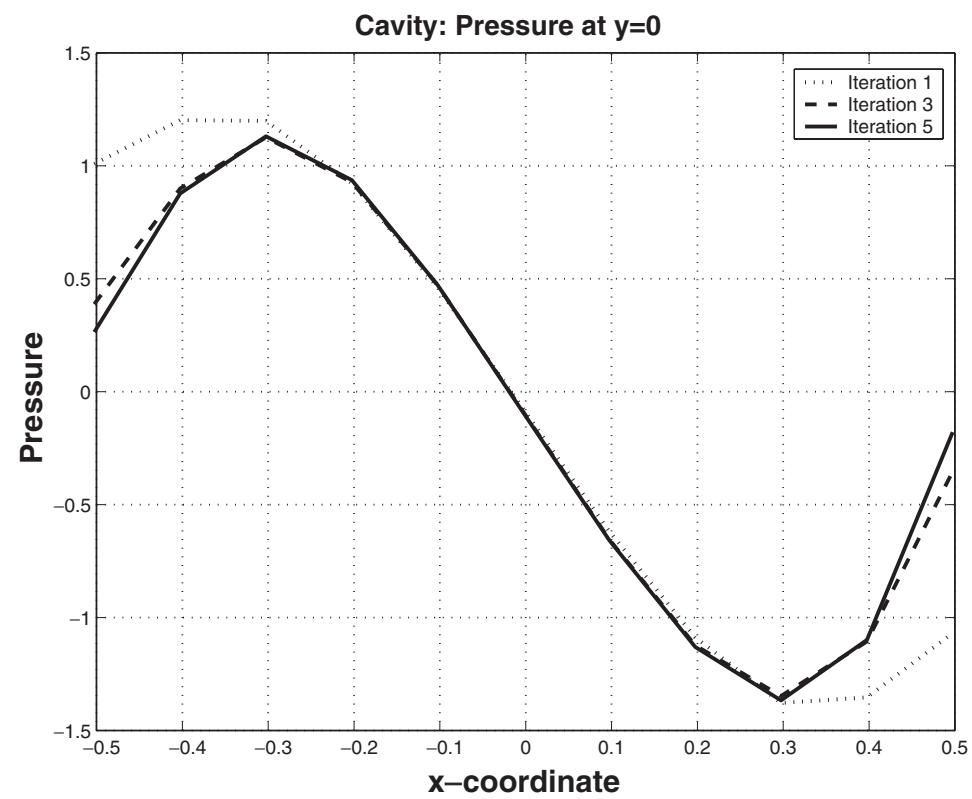

Figure 5. Pressure on horizontal centreline, $10 \times 10$ mesh and iterative solution.

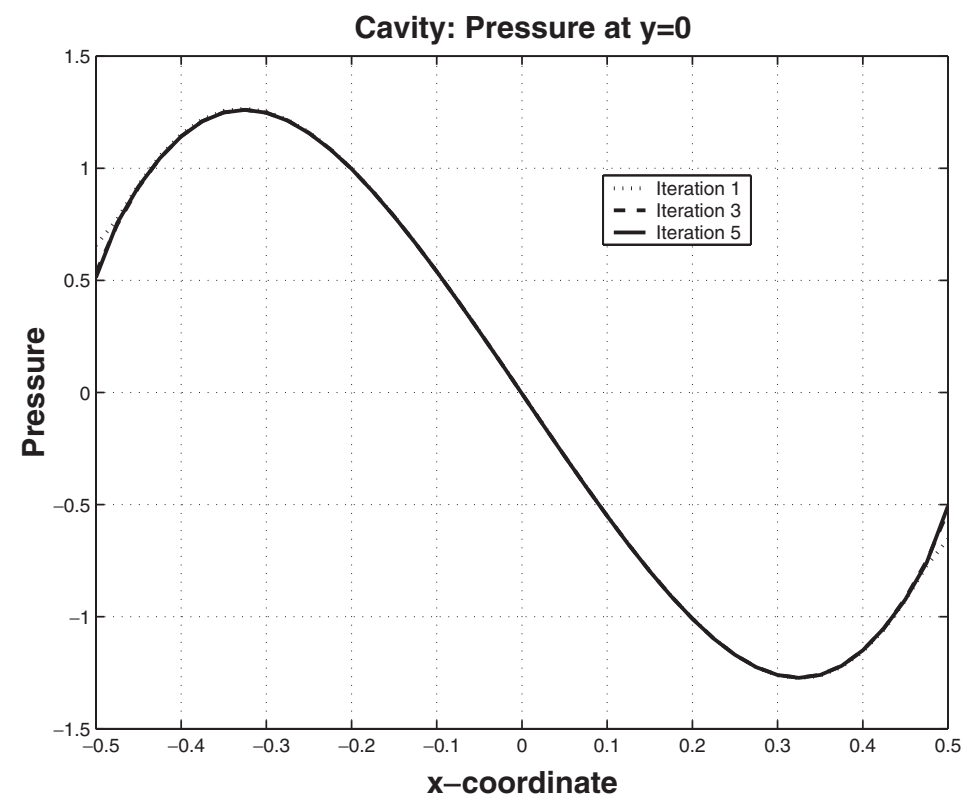

Figure 6. Pressure on horizontal centreline, $40 \times 40$ mesh and iterative solution. 


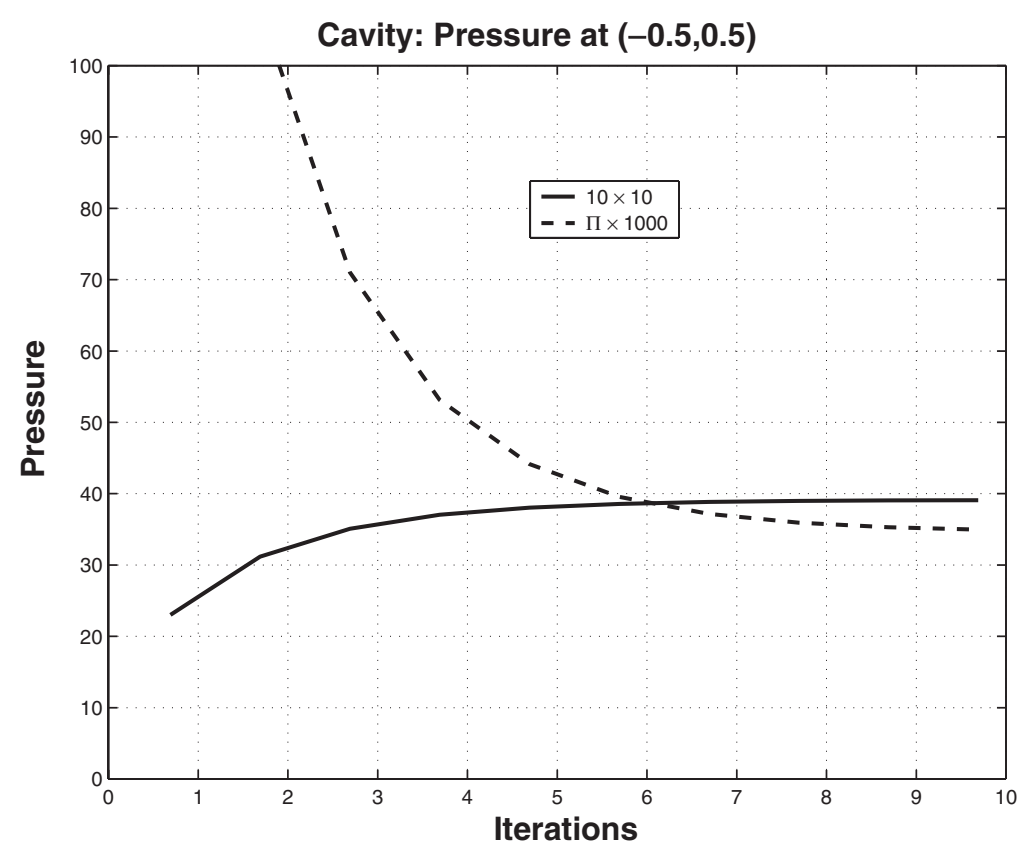

Figure 7. Convergence of pressure $p$ and $\pi=|\pi|$ at upper left corner vs iteration number.
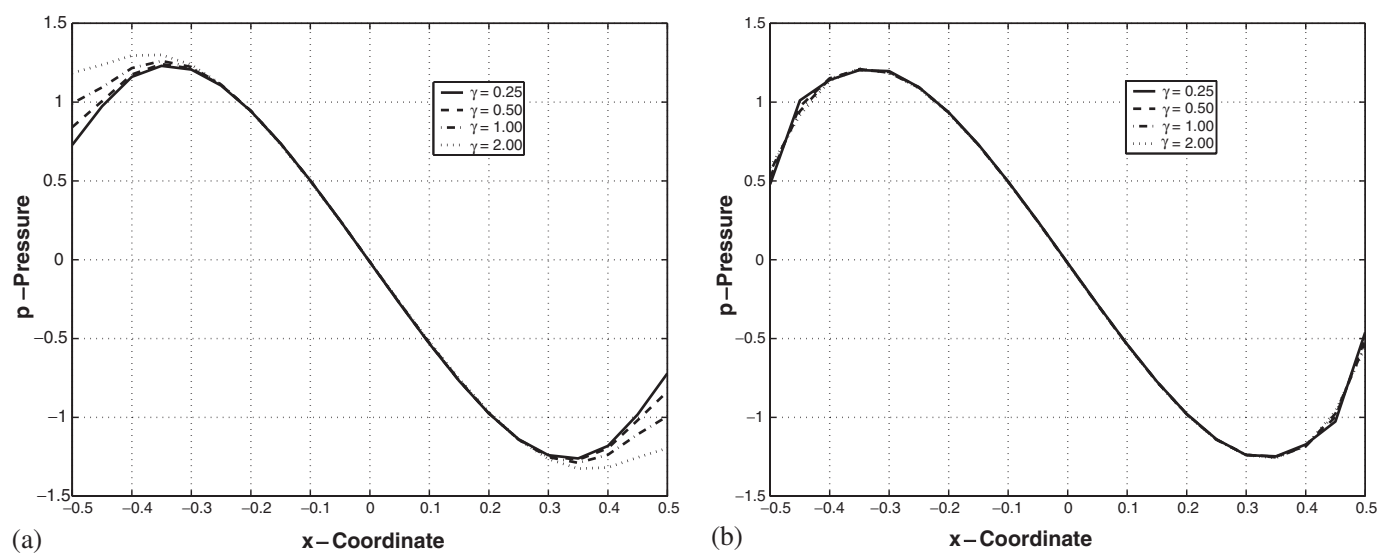

Figure 8. Dependence of pressure on the value of $\gamma=2 G \tau / h^{2}$ for $20 \times 20$ mesh:

(a) solution without $\pi_{i}$; and (b) solution with $\pi_{i}$.

behaviour for the results for $p$ and $\pi=|\pi|$ at the upper left corner is shown in Figure 7 (note that $|\pi|$ is multiplied by 1000 to permit graphical display).

Figure 8 shows the sensitivity of the pressure at the mid-height for different values of the parameter $\gamma$. It is evident that much less dependence on this value results from the addition of the added $\pi_{i}$ stabilization terms. 

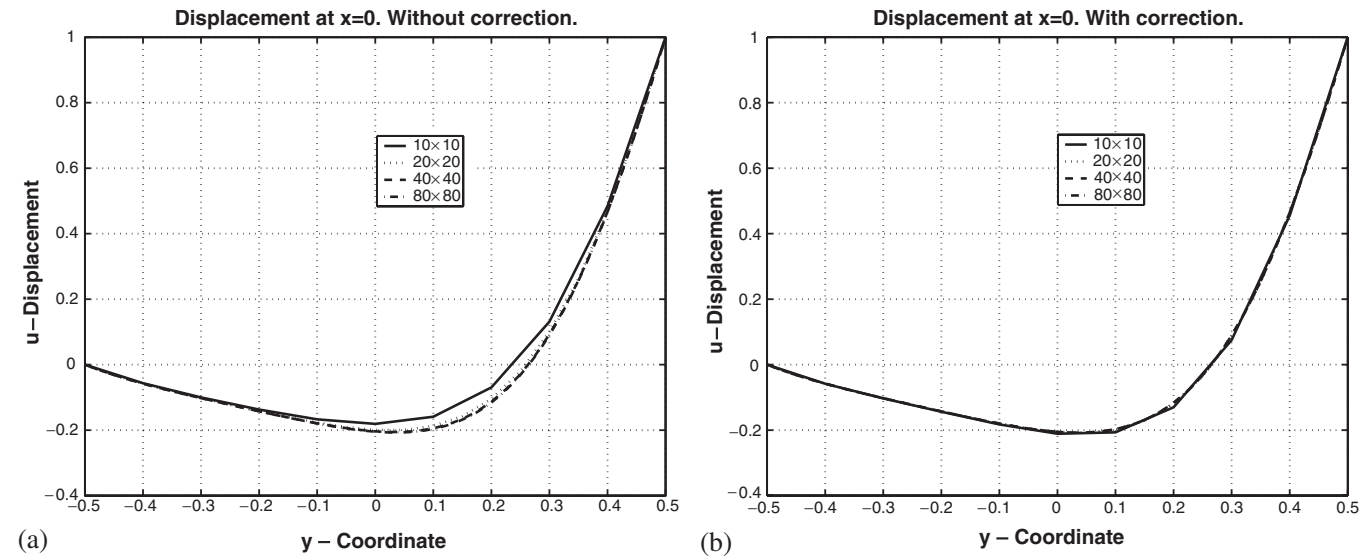

Figure 9. Dependence of $u$-displacement at $x=0$ with mesh subdivision:

(a) solution without $\pi_{i}$; and (b) solution with $\pi_{i}$.
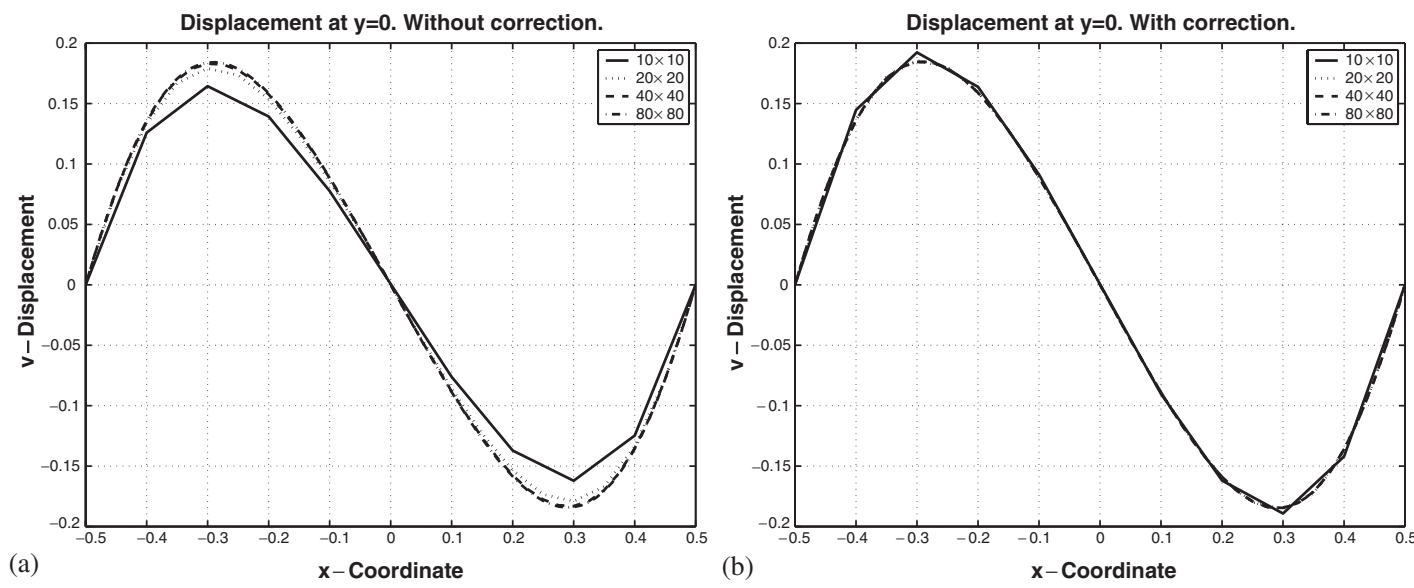

Figure 10. Dependence of $v$-displacement at $y=0$ with mesh subdivision:

(a) solution without $\pi_{i}$; and (b) solution with $\pi_{i}$.

Finally, in Figures 9-11 the convergence of the centreline displacements and pressure is presented for different uniform mesh divisions with 10, 20, 40 and 80 elements per side. Again it is evident that much less sensitivity results from the addition of the $\pi_{i}$ terms.

\subsection{Impact of a cylindrical bar}

The first dynamic problem analysed is the impact of a cylindrical bar with initial velocity of $227 \mathrm{~m} / \mathrm{s}$ into a rigid wall. The bar has an initial length of $32.4 \mathrm{~mm}$ and an initial radius of $3.2 \mathrm{~mm}$. Material properties of the bar are typical of copper: density $\rho=8930 \mathrm{~kg} / \mathrm{m}^{3}$, Young's modulus $E=1.17 \times 10^{5} \mathrm{MPa}$, Poisson's ratio $v=0.35$, initial yield stress $\sigma_{\mathrm{Y}}=400 \mathrm{MPa}$ and hardening modulus $H=100 \mathrm{MPa}$. The period of $80 \mu$ s has been analysed. 

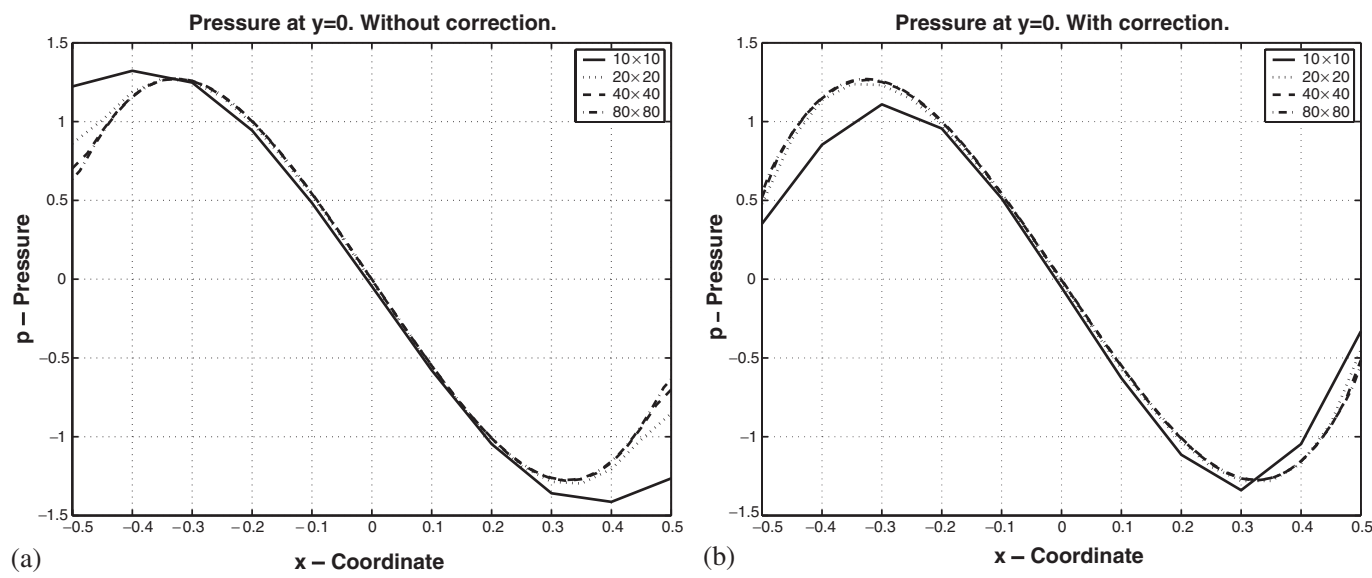

Figure 11. Dependence of pressure at $y=0$ with mesh subdivision:

(a) solution without $\pi_{i}$; and (b) solution with $\pi_{i}$.

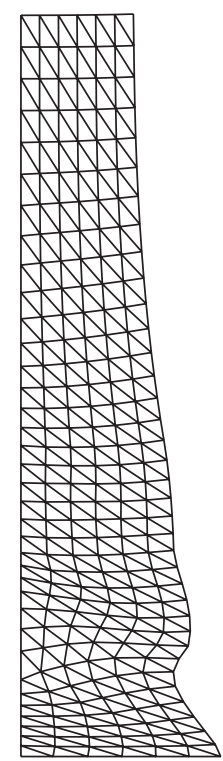

(a)

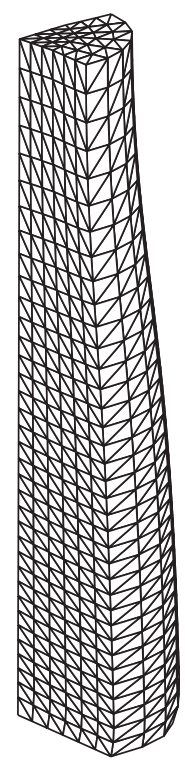

(b)

Figure 12. Final deformed mesh for standard displacement solution with locking: (a) 2D solution using axisymmetric triangular elements; and (b) 3D solution using tetrahedra elements.

Figure 12 shows $2 \mathrm{D}$ and 3D locking solutions using linear triangles and tetrahedra with the standard displacement formulation. Figure 13 shows the numerical results for the pressure and effective plastic strain distribution obtained using a mesh of 216 four node quadrilaterals (259 nodes) with a standard explicit mixed velocity-pressure formulation [1]. Figure 14 shows the results obtained with a mesh of 432 linear triangles (259 nodes) and the proposed semi-explicit 


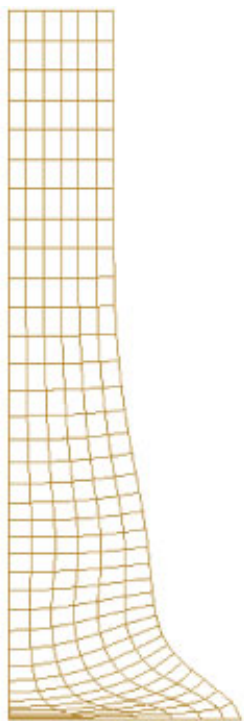

(a)

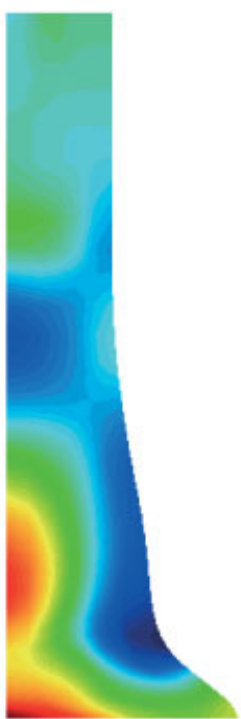

(b)

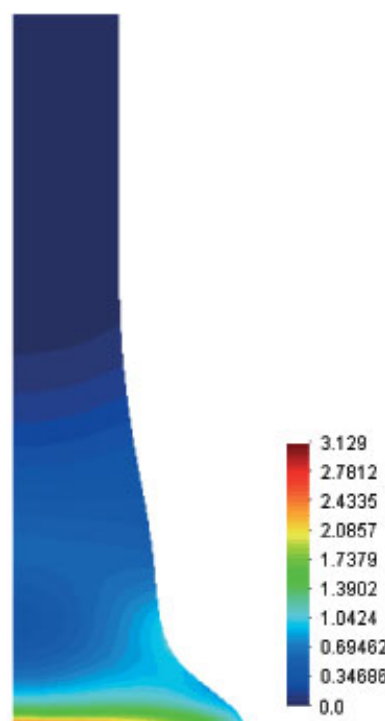

(c)

Figure 13. 2D explicit quasi-incompressible solution using four-node quadrilaterals and a mixed formulation: (a) deformed mesh; (b) pressure distribution; and (c) effective plastic distribution.

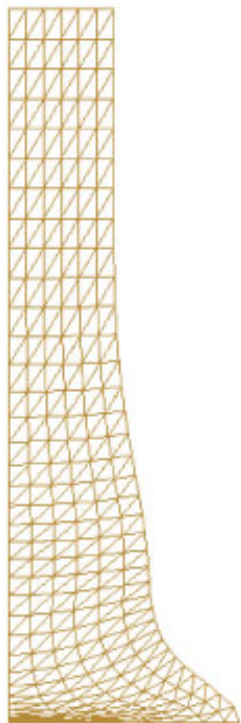

(a)

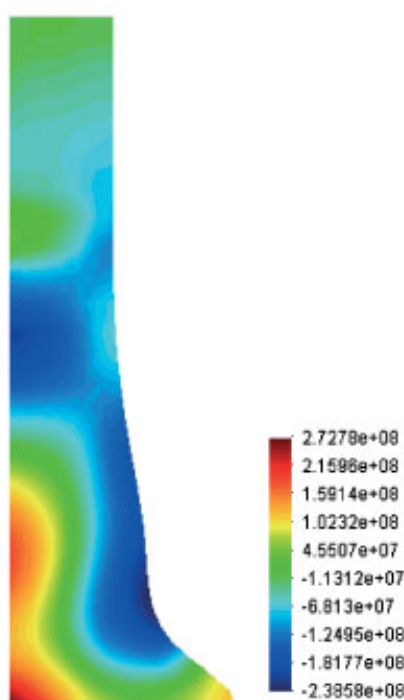

(b)

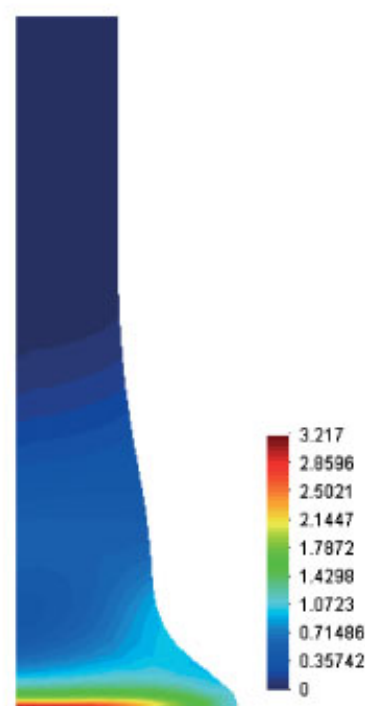

(c)

Figure 14. 2D semi-explicit solution using the FIC formulation: (a) deformed mesh; (b) pressure distribution; and (c) effective plastic distribution. 


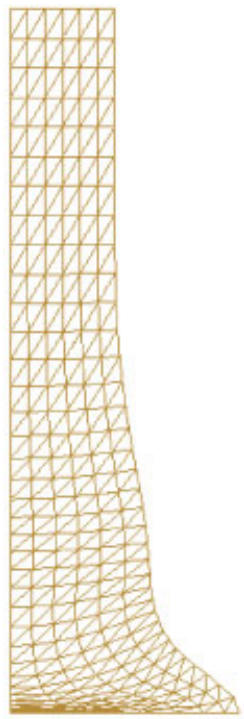

(a)

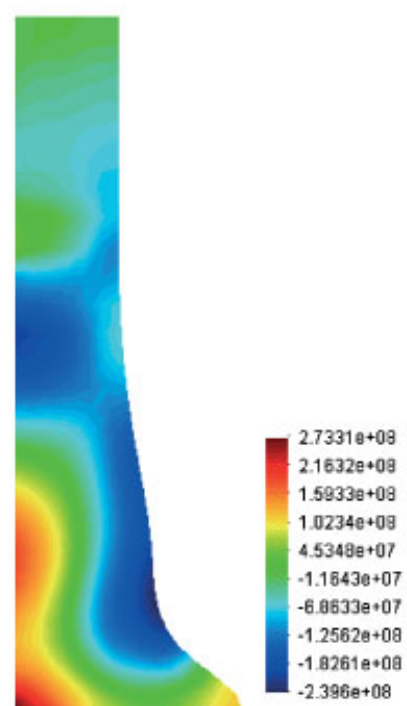

(b)

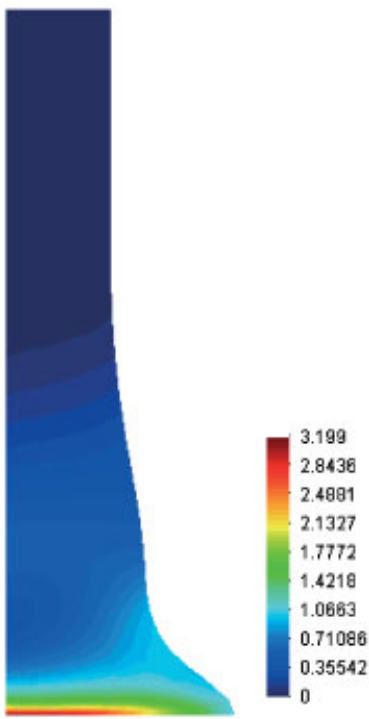

(c)

Figure 15. 2D fully explicit solution using the FIC formulation: (a) deformed mesh; (b) pressure distribution; and (c) effective plastic distribution.

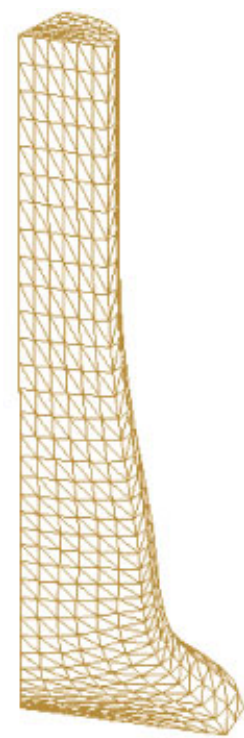

(a)

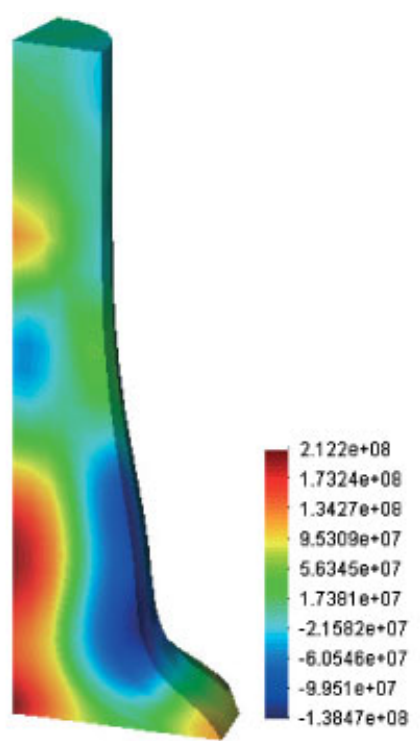

(b)

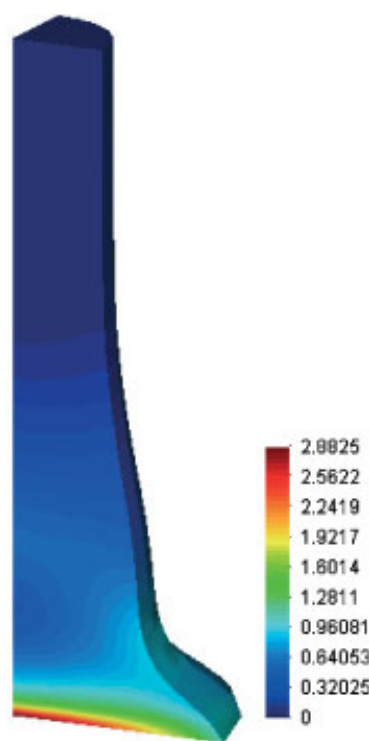

(c)

Figure 16. 3D explicit using the FIC formulation: (a) deformed mesh; (b) pressure distribution; and (c) effective plastic distribution. 


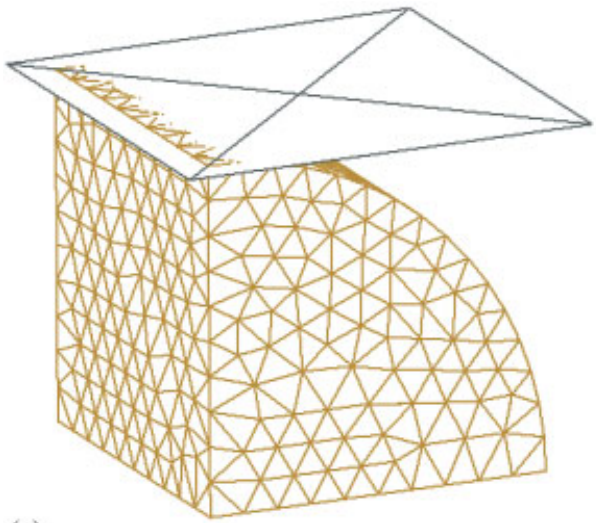

(a)

Figure 17. Sidepressing of a cylinder: (a) initial tetrahedral mesh; and (b) initial hexahedral mesh.

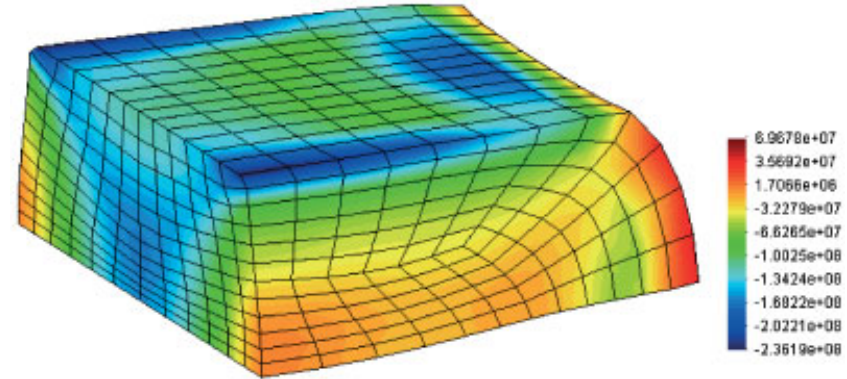

(a)

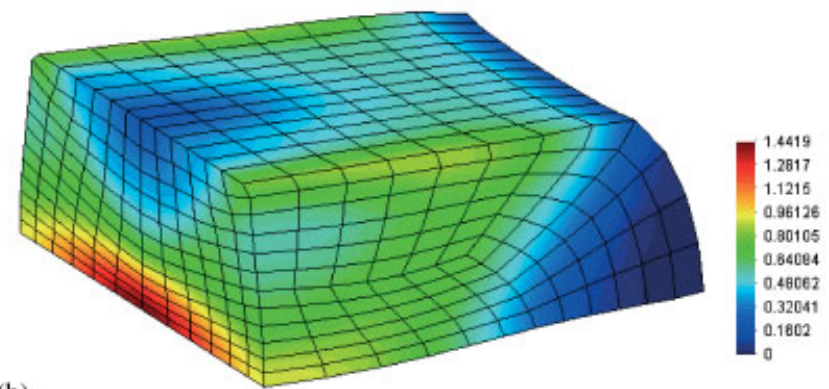

(b)

Figure 18. Sidepressing of a cylinder-results obtained using mixed formulation: (a) pressure distribution; and (b) effective plastic distribution.

algorithm. The initial and final time steps for this case were $0.76 \times 10^{-7}$ and $0.49 \times 10^{-8}$, respectively. Figure 15 shows very similar results obtained using a fully explicit formulation at a considerable smaller storage and computing cost (the time steps for this case were the same as for the semi-explicit solution). 


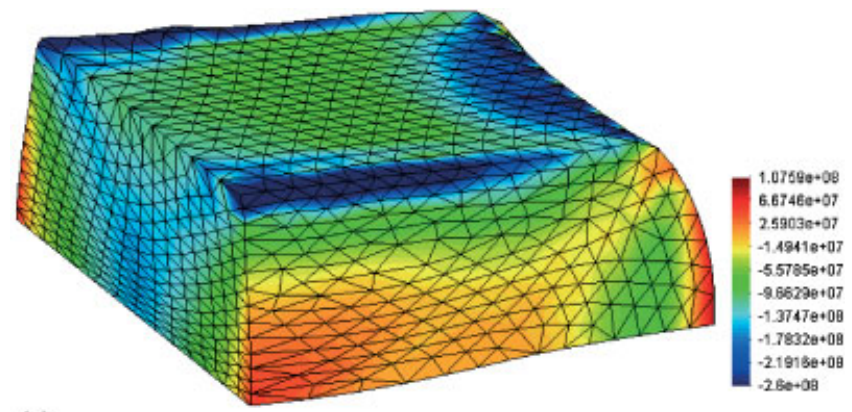

(a)

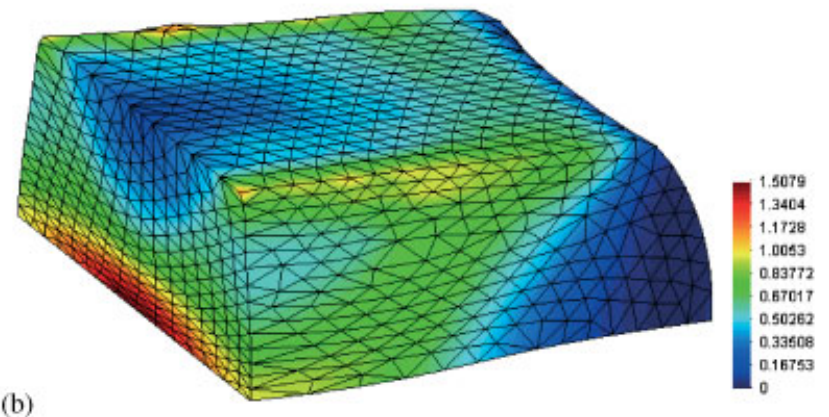

Figure 19. Sidepressing of a cylinder-results obtained with the FIC algorithm for mesh of 22186 elements: (a) pressure distribution; and (b) effective plastic distribution $\left(\tau=(\Delta t)^{2} / \rho\right)$.

Note that values for the equivalent plastic strain obtained with the FIC method coincide well with those given by the mixed formulation. The pressure values also coincide reasonably well in the compression zone, although some differences are found in the peak pressure values induced by tensile (negative) stresses. These differences also occur for solutions obtained with linear triangles and the CBS method $[7,11]$.

Finally, Figure 16 shows the analysis of the same problem using a mesh of 5832 linear tetrahedra (1369 nodes) and the fully explicit formulation. Good stable results are again obtained. This shows that the explicit formulation can be effectively used to solve non-linear dynamic problems of this type.

\subsection{Sidepressing of a cylinder}

A cylinder $100 \mathrm{~mm}$ long with a radius of $100 \mathrm{~mm}$ is subjected to sidepressing between two plane dies. It is compressed up to $100 \mathrm{~mm}$. The material properties are the following: $E=$ $217 \mathrm{GPa}, v=0.3, \rho=7830 \mathrm{~kg} / \mathrm{m}^{3}, \sigma_{0}=170 \mathrm{MPa}, H=30 \mathrm{MPa}$, friction coefficient $=0.2$. The die velocity is assumed to be $2 \mathrm{~m} / \mathrm{s}$. A quarter of a cylinder was discretized using two meshes of 22186 linear tetrahedra (4369 nodes) and 1296 hexahedra (1641 nodes) as shown in Figure 17. Figure 18 shows the numerical results obtained with the four-node hexahedral mesh and an explicit mixed velocity-pressure formulation. Figure 19 shows the pressure and effective plastic strain distributions obtained with the mesh of linear tetrahedra and the fully explicit 
algorithm presented. The maximum and minimum time steps for this solution were $0.5 \times 10^{-5}$ and $0.25 \times 10^{-5}$, respectively. Good agreement between the results obtained with the FIC and the mixed formulation is achieved.

Very similar results were obtained with the linear tetrahedral mesh using the more expensive semi-explicit scheme.

\section{CONCLUSIONS}

The FIC approach is a natural procedure for deriving stabilized finite element methods using equal order interpolation for displacements and pressure for analysis of quasi and fully incompressible solid mechanics problems. The use of projected pressure gradient variables ensures the consistency of the residual terms in the stabilized equation for the pressure and also improves the accuracy of the numerical solution.

When combined with a transient dynamic scheme the FIC formulation provides straightforward semi-implicit and explicit schemes for analysis of non-linear dynamic problems typical of impact and crashworthiness problems and forming processes, among others. The accuracy of the numerical solution can probably be improved by a more rigorous evaluation of the intrinsic time parameter.

\section{ACKNOWLEDGEMENTS}

The authors are thankful to Profs S. Idelhson and C. Felippa for many useful discussions.

\section{REFERENCES}

1. Zienkiewicz OC, Taylor RL. The Finite Element Method. The Basis, (V edn). vol. 1. Butterworth-Heinemann: Oxford, 2000.

2. Babuška I. The finite element method with Lagrangian multipliers. Numerische Mathematik 1973; 20:179-192.

3. Brezzi F. On the existence, uniqueness and approximation of saddle-point problems arising from Lagrange multipliers. Rev. Française d'Automatique Inform. Rech. Opér., Ser. Rouge Anal. Numér. 1974; 8(R-2):129-151.

4. Zienkiewicz OC, Qu S, Taylor RL, Nakazawa S. The patch test for mixed formulations. International Journal for Numerical Methods in Engineering 1986; 23:1873-1883.

5. Zienkiewicz OC, Taylor RL. The finite element patch test revisited: a computer test for convergence, validation and error estimates. Computer Methods in Applied Mechanics and Engineering 1997; 149:523-544.

6. Brezzi F, Pitkäranta J. On the stabilization of finite element approximations of the Stokes problem. In Efficient Solution of Elliptic Problems, Notes on Numerical Fluid Mechanics, Hackbusch W (ed.), vol. 10. Vieweg: Wiesbaden, 1984.

7. Zienkiewicz OC, Rojek J, Taylor RL, Pastor M. Triangles and tetrahedra in explicit dynamic codes for solids. International Journal for Numerical Methods in Engineering 1998; 43:565-583.

8. Zienkiewicz OC, Codina R. A general algorithm for compressible and incompressible flow-Part I. The split, characteristic-based scheme. International Journal for Numerical Methods in Fluids 1995; 20:869-885.

9. Zienkiewicz OC, Morgan K, Satya Sai BVK, Codina R, Vazquez M. A general algorithm for compressible and incompressible flow. Part II. Tests on the explicit form. International Journal for Numerical Methods in Fluids 1995; 20:887-913.

10. Zienkiewicz OC, Taylor RL. The Finite Element Method. Solid Mechanics. (V edn), vol. 3. ButterworthHeinemann: Oxford, 2000.

11. Rojek J, Zienkiewicz OC, Oñate E, Taylor RL. Simulation of metal forming using new formulation of triangular and tetrahedral elements. In 8th International Conference on Metal Forming 2000, Kraków, Poland, Balkema: Rotterdam, 2000. 
12. Hughes TJR, Franca LP, Hulbert GM. A new finite element formulation for computational fluid dynamics: VIII. The Galerkin/least-squares method for advective-diffusive equations. Computer Methods in Applied Mechanics and Engineering 1989; 73:173-189.

13. Bonet J, Marriot H, Hassan O. Stability and comparison of different linear tetrahedral formulations for nearly incompressible explicit dynamic applications. International Journal for Numerical Methods in Engineering 2001; 50:119-133.

14. Bonet J, Marriot H, Hassan O. An average nodal deformation tetrahedron for large strain explicitly dynamic applications. Communications in Numerical Methods in Engineering 2001; 17:551-561.

15. Codina R, Blasco B. Stabilised finite element method for transient Navier-Stokes equations based on pressure gradient projection. Computer Methods in Applied Mechanics and Engineering 2000; 182:287-300.

16. Codina R. Stabilisation of incompressibility and convection through orthogonal sub-scales in finite element methods. Computer Methods in Applied Mechanics and Engineering 2000; 190:1579-1599.

17. Chiumenti M, Valverde Q, Agelet de Saracibar C, Cervera M. A stabilized formulation for incompressible elasticity using linear displacement and pressure interpolations. Computer Methods in Applied Mechanics and Engineering 2002; 191:5253-5264.

18. Chiumenti M, Valverde Q, Agelet de Saracibar C, Cervera M. A stabilized formulation for incompressible plasticity using linear triangles and tetrahedra. International Journal of Plasticity, 2002, submitted.

19. Oñate E. Derivation of stabilized equations for advective-diffusive transport and fluid flow problems. Computer Methods in Applied Mechanics and Engineering 1998; 151:233-267.

20. Oñate E. A stabilized finite element method for incompressible flows using a finite increment calculus formulation. Computer Methods in Applied Mechanics and Engineering 2000; 182:355-370.

21. Oñate E, García J, Idelhson S. Computation of the stabilization parameter for the finite element solution of advective-diffusive problems. International Journal for Numerical Methods in Fluids 1997; 25:1385-1407.

22. Oñate E, García J, Idelhson S. An alpha-adaptive approach for stabilized finite element solution of advectivediffusive problems. In New Advances in Adaptive Computer Methods in Mechanics, Ladeveze P, Oden JT (eds). Elsevier: Amsterdam, 1998.

23. Oñate E, Manzan M. A general procedure for deriving stabilized space-time finite element methods for advective-diffusive problems. International Journal for Numerical Methods in Fluids 1999; 31:203-221.

24. Oñate E, García J. A finite element method for fluid-structure interaction with surface waves using a finite calculus formulation. Computer Methods in Applied Mechanics and Engineering 2001; 191(6-7):635-660.

25. García J, Oñate E. An unstructured finite element solver for ship hydrodynamic problems. Journal of Applied Mechanics 2003; 70:18-26.

26. Oñate E, Sacco C, Idelhson S. A finite point method for incompressible flow problems. Computing and Visualisation in Science 2000; 3:67-75.

27. Oñate E, Rojek J, Taylor RL, Zienkiewicz OC. Linear triangles and tetrahedra for incompressible problem using a finite calculus formulation. In Proceedings of European Conference on Computational Mechanics, Cracow, Poland, 2001, on CD-ROM.

28. Oñate E, Taylor RL, Zienkiewicz OC, Rojek J. A residual correction method based on finite calculus. Engineering Computations 2003; 20:629-658.

29. Felippa CA. Equation modification methods. Personal communication, CIMNE, Barcelona, 2002.

30. Oñate E, Felippa CA. Variational formulations of the finite calculus equations in solid mechanics. Research Report CIMNE IT 416, Barcelona, January 2004.

31. Brooks A, Hughes TJR. Streamline upwind/Petrov-Galerkin formulation for convection dominated flows with particular emphasis on the incompressible Navier-Stokes equations. Computer Methods in Applied Mechanics and Engineering 1982; 32:199-259.

32. Hughes TJR, Mallet M. A new finite element formulations for computational fluid dynamics: III. The generalised streamline operator for multidimensional advective-diffusive systems. Computer Methods in Applied Mechanics and Engineering 1986; 58:305-328.

33. Codina R, Zienkiewicz OC. CBS versus GLS stabilization of the incompressible Navier-Stokes equations and the role of the time step as stabilization parameter. Communications in Numerical Methods in Engineering 2002; 18:99-112.

34. Cervera M, Chiumenti M, Valverde Q, Agelet de Saracibar C. Mixed linear/linear simplicial elements for incompressible elasticity and plasticity. Computer Methods in Applied Mechanics and Engineering 2003; 192:5249-5263. 